\title{
Sequential voting and agenda manipulation
}

\author{
SALVADOR BARBERÀ \\ MOVE, Universitat Autònoma de Barcelona and Barcelona GSE \\ ANKe Gerber \\ Department of Economics, University of Hamburg
}

\begin{abstract}
We study the possibilities for agenda manipulation under strategic voting for two prominent sequential voting procedures: the amendment procedure and the successive procedure. We show that a well known result for tournaments, namely that the successive procedure is (weakly) more manipulable than the amendment procedure at any given preference profile, extends to arbitrary majority quotas. Moreover, our characterizations of the attainable outcomes for arbitrary quotas allow us to compare the possibilities for manipulation across different quotas. It turns out that the simple majority quota maximizes the domain of preference profiles for which neither procedure is manipulable, but at the same time neither the simple majority quota nor any other quota uniformly minimizes the scope of manipulation once this becomes possible. Hence, quite surprisingly, simple majority voting is not necessarily the optimal choice of a society that is concerned about agenda manipulation.
\end{abstract}

KEYWords. Sequential voting, agendas, manipulation.

JEL CLASSIFICATION. C72, D02, D71, D72.

\section{INTRODUCTION}

Many societies and institutions, when choosing among alternatives, resort to sequential (multistage) decision procedures whereby different voters can determine, in a sequence of different steps, which alternatives are definitely out and which ones retain a chance to be considered again, until one of them is definitely selected. In this paper we study two families of classical methods of that sort- the amendment and the successive

Salvador Barberà: salvador . barbera@uab . cat

Anke Gerber: anke.gerber@wiso . uni-hamburg. de

The authors thank two anonymous referees, John Duggan, Matthew Jackson, Inés Macho-Stadler, Hervé Moulin, and seminar audiences at CORE, the 12th Meeting of the Society for Social Choice and Welfare, the 9th Tinbergen Institute Conference, the Workshop on Game Theory in Honor of Marilda Sotomayor, the SITE workshop on the Dynamics of Collective Decision Making, and the IDGP 2015 Workshop, in particular Antoine Loeper and Roger Myerson for valuable comments. Salvador Barberà acknowledges support from the Spanish Ministry of Economy and Competitiveness through the Severo Ochoa Programme for Centers of Excellence in R\&D (SEV-2011-0075), through Grants “Consolidated Group-C” ECO2008-04756 and FEDER, and ECO2014-53051-P, and from the Generalitat de Catalunya through Grants SGR2009-0419 and SGR2014-515. Anke Gerber acknowledges the financial support of MOVE for a research stay at the Universitat Autònoma de Barcelona.

Copyright () 2017 The Authors. Theoretical Economics. The Econometric Society. Licensed under the Creative Commons Attribution-NonCommercial License 3.0. Available at http: //econtheory . org.

DOI: $10.3982 / T E 2118$ 
procedures-both of which are used extensively in many parts of the world. ${ }^{1}$ Our focus will be on the strategic behavior of voters and also of a special agent, to be called the agenda setter, in societies that use these methods.

It is known since ancient times ${ }^{2}$ that the order in which different alternatives are considered along a sequential decision procedure can affect the final choice that a given society may reach, even if the preferences of its members stay the same. Therefore, setting the agenda is a very influential decision, and whoever controls the order of vote often has the possibility to engage in agenda manipulation, that is, to determine the outcome of the choice process. ${ }^{3}$ That power is not absolute, however, since there may be cases where any agenda would lead to the same outcome, as long as the rest of the features defining a rule remain unchanged, and other cases where the range of choices that may be obtained is limited to some subset of all possible alternatives. As already announced, in this paper we analyze the extent to which an agenda setter could choose among several outcomes, and we determine what these can be as a function of the voters' preferences and strategies. Specifically, we assume that voters themselves, given any agenda, will act strategically in a precise way to be described shortly. ${ }^{4}$ The results of our line of analysis are well known for the special case of tournaments, for which Miller (1977) showed that the set of alternatives that are attainable by the successive procedure coincide with the top cycle and for which Banks (1985) provided a characterization of the attainable set for the amendment procedure that became known as the Banks set. However, to the best of our knowledge we are the first to provide characterizations of the sets of attainable alternatives for all possible majority quotas applied to the amendment and the successive voting procedures, including all possible supermajority and submajority quotas. ${ }^{5}$ Our characterizations differ from those of Miller (1977) and Banks (1985) and hence are not straightforward extensions from the case of tournaments to arbitrary quotas. Moreover, our general characterization results allow us to compare the power of the agenda setter across different quotas, which is relevant for institutional design.

The exact characteristics of a sequential voting rule are determined by combining several ingredients, the first of which is what we can call a tree form, which determines

\footnotetext{
${ }^{1}$ Precise definitions of these rules are provided in Section 2. These rules were named by Farquharson (1969) and then studied by Miller $(1977,1980)$ in the special but important case where decisions are made by simple majority. A recent axiomatic characterization is in Apesteguia et al. (2014). The relevance of these methods in parliamentary practice and their use in different countries is discussed in Rasch (2000).

${ }^{2}$ See the Letter to Titus Aristo by Pliny the Younger (A.D. 105) reproduced in McLean and Urken (1995). Farquharson's path-breaking book (1969) uses that letter extensively for motivation and analysis.

${ }^{3}$ We concentrate on manipulations that involve changes in the order of vote, while keeping the same set of alternatives. Other forms of agenda manipulation involve the addition of new items to the agenda or the removal of some alternatives. This has been studied, among others, by Dutta et al. (2004) and Duggan (2006).

${ }^{4}$ Under some binary rules, like the amendment procedure, it is clear what it would mean to act nonstrategically. Under other rules, like the successive procedure, that notion is not as obvious. Whenever the notion of nonstrategic behavior is well defined, it is clear that an agenda setter could also derive advantages from choosing the agenda in many cases.

${ }^{5}$ Apart from the theoretical motivation to deliver a comprehensive analysis that covers all majority quotas, submajority quotas are also empirically relevant, because there are a number of institutions that actually use submajority rules (see Vermeule 2005).
} 
two aspects of the sequential process. One aspect is the number and the nature of actions that agents can take at any node, starting from an initial node, until a terminal node is reached at the end of each path. But since one and only one alternative will eventually be attached to each terminal node, so as to define trees, a tree form is also defined by any restriction that may be imposed on the possible assignment of the same alternative to different terminal nodes. The two families of procedures we study here are based on binary tree forms, where each nonterminal node has two successors. The second ingredient defining a sequential rule is the agenda, that is, the specific assignment of alternatives to terminal nodes, respecting the restrictions imposed by the tree form. That assignment determines what choices will be made by society after following the possible path that leads to each terminal node. In all the cases we study, an agenda is just an order over the alternatives, because we provide specific and unique rules that translate each possible order into a unique admissible assignment of alternatives to the terminal nodes of the tree forms that we consider. A tree is then given by a tree form and by an agenda. Now, to turn a tree into a sequential voting rule, we must specify how the different members of a voting body will influence the choice of paths along the tree. Since we are working with binary trees, and we want to consider methods that treat all agents on the same footing, we consider as possible methods all those that are defined by a quota $q$, with $q$ between 1 and the number of voters. When confronted with two choices at any node, society will move to a prespecified successor of that node if at least $q$ people vote for it, and otherwise will take the opposite path.

A sequential voting rule will thus be fully specified once we have a tree and a quota. Of course, a voting rule is defined independently of the preferences that may be held by different agents regarding the alternatives. It sets the rules through which agents will be able to contribute to the social decision. But to study the behavior of different agents under these rules, we need to know what their preferences will be. And then, given a profile of preferences, we will have all the elements to study the strategic behavior of those agents. A tree and a quota then provide a game form, and when we add to them a preference profile we have a game.

Although our motivation is to study the strategic behavior of voters under these sequential rules, it turns out that most of our analysis can be carried out by just knowing a dominance relation among alternatives that generalizes the notion of a tournament, and that can be used to represent the preferences of society. Whereas a tournament is any complete and asymmetric relation over alternatives, the binary relations generated by comparing alternatives according to quotas different than simple majority give rise to relations that may fail one of these two properties. ${ }^{6}$ Moreover, some relations that are either complete but not asymmetric or asymmetric and not complete may never be obtained as the dominance relation induced by a quota and a preference profile. Yet our main characterization results still hold for this larger class of social preferences. Because of that, our work can also be understood as a natural extension of tournament theory, and the sets we identify can be compared to the different solution sets proposed

\footnotetext{
${ }^{6}$ For supermajority quotas, the binary relation may be incomplete, while for submajority quotas, the relation may violate asymmetry.
} 
for tournaments and for their extensions (Miller 1977 and 1980, Shepsle and Weingast 1984, Banks 1985, Moulin 1986, Banks and Bordes 1988, Laslier 1997).

We first provide characterizations of the unique equilibrium outcomes obtained by iterative elimination of weakly dominated strategies for each of the two families of games we consider. We then use these characterizations to identify the sets of alternatives that could be the outcome of games that share the same tree form and the same rule for choosing among nodes, but differ on the agenda. Comparisons among these sets allow us to discuss the degree of agenda manipulability of different rules in our classes. It turns out that both procedures are nonmanipulable on the same set of preference profiles, namely those profiles for which there exists a (generalized) Condorcet winner, i.e., an alternative that dominates all others and in turn is not dominated. Moreover, if there is no Condorcet winner, then the successive procedure is more vulnerable toward agenda manipulation than the amendment procedure in the following sense: at any preference profile (or, more generally, for any dominance relation), any outcome that can be achieved for some agenda under the amendment procedure can also be achieved by some agenda under the successive procedure, while the reverse is not true in general. While this result was already known for tournaments given the characterizations of Miller (1977) and Banks (1985), we are able to show that it holds for all quotas.

Comparing different quotas under the same sequential voting procedure, we find that the set of preference profiles that do not allow for manipulation is maximized at simple majority voting and is otherwise weakly decreasing (increasing) in the quota for supermajority (submajority) quotas. This gives some support for simple majority voting if the possibility of agenda manipulation is a concern. But if at a given preference profile there are opportunities for agenda manipulation under simple majority voting, then there is no quota that uniformly minimizes the degree of manipulability, neither for the successive nor for the amendment procedure. There are even cases where a submajority quota minimizes the possibilities for manipulation.

The outline of the paper is as follows. In Section 2 we introduce general binary voting games and derive the equilibrium outcome of the voting game for the amendment and the sequential procedures at a given agenda. In Sections 3 and 4 we characterize the set of outcomes that can be obtained by agenda manipulation for the amendment and sequential procedures. In Section 5 we compare the scope of manipulation under the amendment and successive procedures for different quotas. Section 6 discusses the robustness of our rules with respect to some conventions we adopt in their definition. Section 7 concludes.

\section{Sequential binary Voting games}

Let there be a finite set of alternatives $X$ with $\# X \geq 2 .{ }^{7}$ A binary voting tree on $X$ is a tree in which every nonterminal node has exactly two successors—left and right—and to every terminal node an alternative in $X$ is assigned, so that this mapping is onto. ${ }^{8}$

\footnotetext{
${ }^{7}$ The term \# $A$ denotes the number of elements in a finite set $A$.

${ }^{8}$ For purposes of expediency we define trees directly, rather than starting with tree forms as introduced in Section 1. Thus, at this stage the role of agendas is implicit, and is the one suggested in Section 1. It
} 
Formally, we define a binary voting tree on $X$ to be a quadruple $(X, N, \triangleright, \phi)$, such that the following conditions are satisfied.

Condition $1 . N$ is a finite set of nodes.

Condition 2. $\triangleright$ is a binary relation on $N$ that satisfies the following conditions:

(i) There exists a unique $\nu_{0} \in N$ (the initial node) such that

$$
\left\{\nu \mid \nu \in N \text { and } \nu_{0} \triangleright \nu\right\}=\varnothing .
$$

(ii) For all $\nu \in N \backslash\left\{\nu_{0}\right\}$, there exists a unique $\nu^{\prime} \in N$ with $\nu \triangleright \nu^{\prime}$.

(iii) There exists a nonempty subset $T \subset N$ of terminal nodes such that for all $\nu \in T$,

$$
\left\{\nu^{\prime} \mid \nu^{\prime} \in N \text { and } \nu^{\prime} \triangleright \nu\right\}=\varnothing .
$$

(iv) For all $\nu \in N \backslash T,\left\{\nu^{\prime} \mid \nu^{\prime} \triangleright \nu\right\}=\{l(\nu), r(\nu)\} .^{9}$

Condition 3. $\phi: T \rightarrow X$ is an onto function assigning to each terminal node a unique alternative in $X$.

If $\nu \triangleright \nu^{\prime}$ for $\nu, \nu^{\prime} \in N$, then we call $\nu$ a successor of $\nu^{\prime}$ and call $\nu^{\prime}$ a predecessor of $\nu$. A nonterminal node of a binary voting tree on $X$ is called a decision node.

Let there be $n$ voters. Each voter $i$ 's strategy is a function $\sigma_{i}: N \backslash T \rightarrow N$ such that $\sigma_{i}(\nu) \in\{l(\nu), r(\nu)\}$ for all $\nu \in N \backslash T$. That is, a strategy indicates which of the two possible successor nodes the voter will vote for once he/she arrives at node $\nu$. To determine the outcome resulting from any $n$-tuple of strategies, we must describe which of the two nodes $l(\nu)$ or $r(\nu)$ will be selected as a continuation, given that $\nu$ was reached. To do that, consider any quota $q$ between 1 and $n$, which will be used to determine which of the two successors is a $q$ winner. When one of the two nodes obtains $q$ votes and the other does not, the node most voted for is declared the $q$ winner. Exceptionally, when $n$ is odd and $q=(n+1) / 2$, it must always be that one and only one of the two competing nodes is a winner in that sense. Otherwise, there may be some ambiguity left. It may be that $n$ is even, $q=\frac{n}{2}$, and both $l(\nu)$ and $r(\nu)$ obtain $q$ votes. It could also be that $q<\frac{n}{2}$ and both obtain $q$ or more votes or that $q>\frac{n}{2}$ and the votes for none of the two nodes reaches the quota. In all these cases, determining which alternative will confront the following vote, or become the winner, requires the use of a convention. Although these cases are obviously different, we may sometimes follow tradition, referring colloquially to all of them as situations where there is a tie, and call the convention that we adopt regarding which node is favored a tie-breaking rule. We could think of this convention as providing the advantage of being a (temporary) status quo to either $l(v)$ or to $r(v)$. In the main part of this paper, we use the convention that $l(\nu)$ will be the $q$ winner if it gets at least $q$ votes

will become more explicit when we introduce the binary voting games for the amendment and successive procedures below.

${ }^{9}$ Since every decision node is assumed to have exactly two successors, we follow Austen-Smith and Banks (2005) and label the successors of every $\nu \in N \backslash T$ as $l(\nu)$ (left successor), and $r(\nu)$ (right successor). 
and that $r(\nu)$ is the $q$ winner otherwise. This convention is in agreement with the most usual interpretation of sequential voting methods as representations of legislative voting procedures where the status quo prevails unless some alternative strictly dominates it, and the status quo is the last (right) alternative to appear. But other variants of the model could express the same idea: for example, we could consider that the status quo is the first (left) element in the list and that it takes a $q$ majority to defeat the left alternatives, or we could also switch the roles of right and left alternatives and modify the rules accordingly. As a result, all variants essentially reduce to two: the one we consider in the main text and the alternative formulation that we briefly describe in Section 6. Our main points regarding the manipulability of the different rules are maintained, and are robust to the alternative specifications, with small variations that are spelled out in that penultimate section of the paper.

Now, each quota, along with its associated notion of a $q$-majority winner, allows us to associate a unique terminal node $\nu(\sigma)$ and a unique alternative $\phi(\nu(\sigma)) \in X$ to every $n$-tuple of strategies $\sigma=\left(\sigma_{1}, \ldots, \sigma_{n}\right)$ in a natural manner. At every nonterminal node $\nu$ there is a $q$-majority vote over the successors $l(\nu)$ and $r(\nu)$, such that $l(\nu)$ wins if at least $q$ voters vote in favor of $l(\nu)$, and $r(\nu)$ wins otherwise. If $r(\nu)$ wins, the next $q$-majority vote is over the successors $l(r(\nu))$ and $r(r(\nu))$ of $r(\nu)$, while if $l(\nu)$ wins, the next $q$-majority vote is over the successors $l(l(\nu))$ and $r(l(\nu))$ of $l(\nu)$. The strategy sets associated to a tree structure, along with the outcome function associated to the tree under any quota $q$ define a game form, just short of being a game in the absence of the voters' preferences.

We now turn to these preferences. Every voter $i$ is assumed to have a strict preference ordering $\mathcal{P}_{i}$ over $X$, i.e., $\mathcal{P}_{i}$ is complete (for all $x, y \in X$ with $x \neq y$, it is true that $x \mathcal{P}_{i} y$ or $y \mathcal{P}_{i} x$ ), transitive (for all $x, y, z \in X$, if $x \mathcal{P}_{i} y$ and $y \mathcal{P}_{i} z$, then $x \mathcal{P}_{i} z$ ), and asymmetric (for all $x, y \in X$, if $x \mathcal{P}_{i} y$, then $\left.\neg y \mathcal{P}_{i} x\right)$. Let $\mathcal{P}=\left(\mathcal{P}_{1}, \ldots, \mathcal{P}_{n}\right)$ be the profile of voters' preferences. Now, for any game form associated to a voting tree, its strategy space, and a given quota, we can define a sequential binary voting game $(X, N, \triangleright, \phi, \mathcal{P}, q)$ as the one resulting from endowing agents with a given preference profile.

Since the sequential binary voting games defined above can have very implausible Nash equilibria, where all voters coordinate on the same strategy irrespective of their preferences, we restrict to the class of Nash equilibria in undominated strategies as is common in the literature on voting games. Recall that a normal form game is dominance solvable if all players are indifferent between all strategy profiles that survive the iterative procedure where all weakly dominated strategies of all players are simultaneously eliminated at each stage. An extensive form game (like the sequential binary voting game defined above) is dominance solvable if the corresponding normal form game is dominance solvable. ${ }^{10}$ We will now argue that the sequential binary voting game $(X, N, \triangleright, \phi, \mathcal{P}, q)$ is dominance solvable for all quotas $q$ : first, for every voter $i$ we can eliminate all strategies, where $i$ does not vote for his preferred terminal node at every last decision node, i.e., at every decision node whose successors are two terminal nodes. Observe that given the strict preference ordering $\mathcal{P}_{i}$, voter $i$ is indifferent between two terminal nodes $\nu$ and $\nu^{\prime}$ if and only if $\phi(\nu)=\phi\left(\nu^{\prime}\right)$. Hence, voter $i$ is indifferent between two terminal nodes if and only if all voters $j \neq i$ are indifferent between these

\footnotetext{
${ }^{10}$ In voting theory dominance solvability is also known as sophisticated voting (see Farquharson 1969).
} 
nodes. Thus, conditional on reaching a specific terminal decision node, all strategy profiles surviving the first elimination round are outcome equivalent. Hence, after the first elimination round every voter has well defined preferences over all last decision nodes since all these nodes are associated with a unique outcome (alternative) under the surviving strategy profiles. We continue by eliminating for every voter $i$ all strategies where $i$ does not vote for his preferred successor node at every penultimate decision node, i.e., at every decision node that is a predecessor of two last decision nodes. Again, after this second elimination round every voter has well defined preferences over all penultimate decision nodes and if one voter is indifferent between two penultimate decision nodes, all voters are indifferent. Continuing in this way we finally arrive at the initial node and we eliminate for every voter $i$ all strategies where $i$ does not vote for his preferred successor node. Then all voters are indifferent between all remaining strategy profiles, and all these surviving profiles $\sigma$ lead to the same alternative $\phi(\nu(\sigma)) \in X$, which we call the outcome, $o(X, N, \triangleright, \phi, \mathcal{P}, q)$, of the sequential binary voting game. Hence, we have the following result (cf. McKelvey and Niemi 1978, Moulin 1979, Gretlein 1982, AustenSmith and Banks 2005).

Theorem 2.1. Every sequential binary voting game $(X, N, \triangleright, \phi, \mathcal{P}, q)$ is dominance solvable.

In this paper we will focus on two specific binary voting trees on $X$ that represent two prominent sequential voting procedures: the amendment procedure and the successive procedure. Both procedures start with an agenda, i.e., an ordering $\left(x_{1}, x_{2}, \ldots, x_{m}\right)$ of the alternatives in $X$, where the nontrivial case is when $m \geq 2 .{ }^{11}$

\section{Amendment procedure}

Given an agenda $\left(x_{1}, \ldots, x_{m}\right)$, the binary voting tree $(X, N, \triangleright, \phi)$ for the amendment procedure is such that the first vote is over $x_{1}$ and $x_{2}$, the second vote is over the winner of the first vote and $x_{3}$, the third vote is over the winner of the second vote and $x_{4}$, and so on until all alternatives are exhausted. Figure 1 shows the binary voting tree for the amendment procedure in the case where $m=3$. Observe that the agenda also yields a natural labeling of the two successor nodes of every nonterminal decision node: at every decision node $\nu$ there is a vote over two alternatives, $x_{i}$ and $x_{j}$, where $i<j$. The left successor, $l(\nu)$, then is the node reached if alternative $x_{i}$ wins, and $r(\nu)$ is the node reached if alternative $x_{j}$ wins.

\footnotetext{
${ }^{11}$ We follow Shepsle and Weingast (1984) and Banks (1985) by calling an agenda an ordering of the alternatives. But, unfortunately, the terminology in the related literature is not always the same. Miller (1995) calls a binary voting tree an agenda. Laslier (1997) uses the term agenda to designate a binary voting tree where no alternatives have been assigned to the terminal nodes yet, and he calls the order of alternatives a drawing. Our choice of words tries to reflect the fact that the tree, along with the rules determining how to move from node to node through its branches after each vote, represents the formal procedure, while the agenda refers to the substantial issues at hand and the order in which they are debated. At any rate, terminology is not a problem when it comes to comparing results.
} 


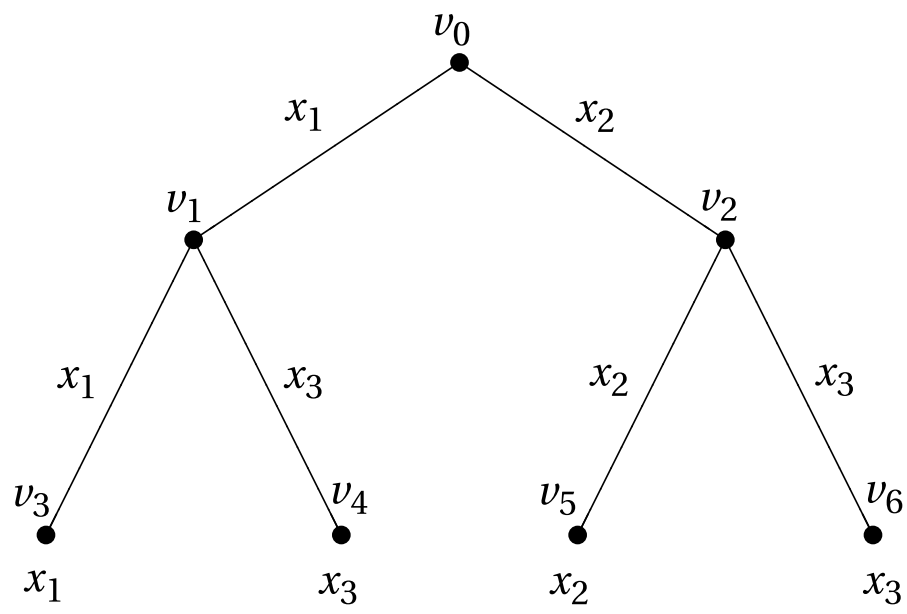

FIGURE 1. Binary voting tree for the amendment procedure with agenda $\left(x_{1}, x_{2}, x_{3}\right)$.

Consider now the sequential binary voting game $(X, N, \triangleright, \phi, \mathcal{P}, q)$ for the amendment procedure. By Theorem 2.1 the game is dominance solvable and we have seen that there is a simple backward induction procedure to derive the unique outcome of the game. To determine this outcome, we let $P$ denote the social preference relation on $X$ under sincere voting with quota $q$, i.e. for all $x, y \in X$,

$$
x P y \quad \Longleftrightarrow \quad \#\left\{i \mid x \mathcal{P}_{i} y\right\} \geq q .
$$

Observe that for a given quota $q, P$ is either complete (if $q \leq\left\lceil\frac{n}{2}\right\rceil$ ) or asymmetric (if $q \geq\left\lfloor\frac{n}{2}\right\rfloor+1$ ) or both. ${ }^{12}$ In the latter case $P$ defines a tournament. ${ }^{13}$ By allowing $P$ to be incomplete or to violate asymmetry we depart from the tournament literature and provide a more general analysis of sequential voting games. Let $o^{A}\left(x_{1}, x_{2}, \ldots, x_{m}\right)$ denote the outcome of the sequential binary voting game for the amendment procedure with a given agenda $\left(x_{1}, \ldots, x_{m}\right)$. Then $o^{A}\left(x_{1}, x_{2}, \ldots, x_{m}\right)$ is inductively defined over the number of alternatives in the agenda as follows.

If $m=1$, then $o^{A}\left(x_{1}\right)=x_{1}$. Let $m \geq 2$ and suppose the outcome has been defined for any agenda with up to $m-1$ alternatives. Consider the agenda $\left(x_{1}, x_{2}, \ldots, x_{m}\right)$. Then

$$
\begin{aligned}
& o^{A}\left(x_{1}, x_{2}, \ldots, x_{m}\right) \\
& \quad= \begin{cases}o^{A}\left(x_{1}, x_{3}, \ldots, x_{m}\right) & \text { if } o^{A}\left(x_{1}, x_{3}, \ldots, x_{m}\right) P o^{A}\left(x_{2}, x_{3}, \ldots, x_{m}\right), \\
o^{A}\left(x_{2}, x_{3}, \ldots, x_{m}\right) & \text { if } \neg o^{A}\left(x_{1}, x_{3}, \ldots, x_{m}\right) P o^{A}\left(x_{2}, x_{3}, \ldots, x_{m}\right) .\end{cases}
\end{aligned}
$$

Note that in (2) we have used the tie-breaking rule introduced previously: $x_{1}$ wins against $x_{2}$ if and only if the ultimate outcome that is reached if we let $x_{1}$ pass, $o^{A}\left(x_{1}, x_{3}, \ldots, x_{m}\right)$, defeats the ultimate outcome $o^{A}\left(x_{2}, x_{3}, \ldots, x_{m}\right)$ that is reached if we

\footnotetext{
${ }^{12}$ Here, $\lfloor c\rfloor(\lceil c\rceil)$ denotes the largest (smallest) integer less (larger) than or equal to $c \in \mathbb{R}$.

${ }^{13}$ This is the case if $n$ is odd and $q=(n+1) / 2$.
} 


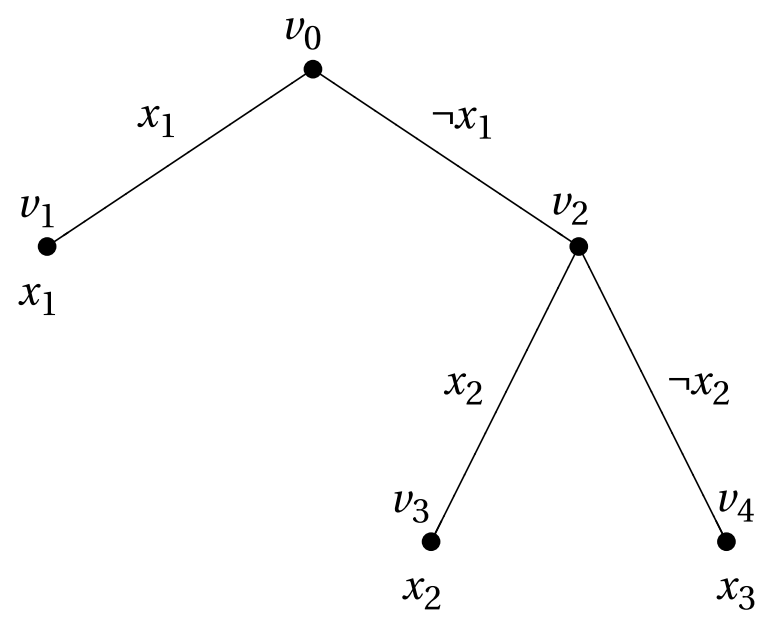

FigURE 2. Binary voting tree for the successive procedure with agenda $\left(x_{1}, x_{2}, x_{3}\right)$.

let $x_{2}$ pass. Hence, in any pairwise vote the tie-breaking rule can be interpreted as attributing to the alternative introduced later the role of a status quo that prevails unless it is defeated by the alternative introduced earlier. ${ }^{14}$

\section{Successive procedure}

Given an agenda $\left(x_{1}, \ldots, x_{m}\right)$, the binary voting tree $(X, N, \triangleright, \phi)$ for the successive procedure is such that the first vote is over the approval of $x_{1}$. If $x_{1}$ is approved, the voting is over and the outcome is $x_{1}$. If $x_{1}$ is rejected, the next vote is over the approval of $x_{2}$. If $x_{2}$ is approved, the voting is over and the outcome is $x_{2}$. Otherwise, if $x_{2}$ is rejected, the next vote is over the approval of $x_{3}$, and so on. If $x_{m-1}$ is rejected, the outcome is $x_{m}$. Figure 2 shows the binary voting tree for the successive procedure in the case where $m=3$. Again, the agenda yields a natural labeling of the two successor nodes of every nonterminal decision node: at every decision node $\nu$ there is a vote over approving or rejecting an alternative $x_{i}$. The left successor, $l(\nu)$, then is the node reached if $x_{i}$ is approved, and $r(\nu)$ is the node reached if $x_{i}$ is rejected.

As for the amendment procedure, we consider the sequential binary voting game $(X, N, \triangleright, \phi, \mathcal{P}, q)$ for the successive procedure. Again, let $P$ be the social preference relation over $X$ as defined in (1). Then the outcome $o^{S}\left(x_{1}, x_{2}, \ldots, x_{m}\right)$ for the successive procedure is inductively defined over the number of alternatives in the agenda as follows.

\footnotetext{
${ }^{14}$ This is in line with the convention in legislative voting to place the status quo bill last in the agenda and before that the alternative bill, the amendment to the alternative bill, and so on. At each stage of the sequential voting, a bill then is replaced by an amended version if and only if the amended version defeats it. We refer the reader to Section 6 for a discussion of the alternative tie-breaking rule where the earlier alternative in an agenda is treated as a status quo.
} 
If $m=1$, then $o^{S}\left(x_{1}\right)=x_{1}$. Let $m \geq 2$ and suppose the outcome has been defined for any agenda with up to $m-1$ alternatives. Consider the agenda $\left(x_{1}, x_{2}, \ldots, x_{m}\right)$. Then

$$
o^{S}\left(x_{1}, x_{2}, \ldots, x_{m}\right)= \begin{cases}x_{1} & \text { if } x_{1} P o^{S}\left(x_{2}, x_{3}, \ldots, x_{m}\right), \\ o^{S}\left(x_{2}, x_{3}, \ldots, x_{m}\right) & \text { if } \neg x_{1} P o^{S}\left(x_{2}, x_{3}, \ldots, x_{m}\right) .\end{cases}
$$

Again note that in (3) we have used the tie-breaking rule introduced before, according to which $x_{1}$ is approved if and only if $x_{1}$ defeats the ultimate outcome $o^{S}\left(x_{2}, x_{3}, \ldots, x_{m}\right)$ that is reached if $x_{1}$ is rejected. ${ }^{15}$

The inductive definitions of $o^{A}\left(x_{1}, x_{2}, \ldots, x_{m}\right)$ and $o^{S}\left(x_{1}, x_{2}, \ldots, x_{m}\right)$ for the amendment and successive procedure in (2) and (3) reveal that the outcome of an agenda only depends on the social preference relation $P$ and is invariant with respect to changes in the individual preferences $\mathcal{P}_{i}$ that leave $P$ unchanged. Hence, in the following we will consider the general case, where society makes binary choices according to an arbitrary binary relation $P$ on $X$, which is not necessarily derived from majority voting with quota $q$. We refer to $P$ as a dominance relation and continue to use the term social preference relation if $P$ is derived from $q$-majority voting. If we assume that society is rational in the sense that at every decision node in the binary voting tree it chooses the successor node that ultimately leads to the alternative that is preferred according to $P$, then the outcome of an agenda for the amendment and successive procedures is still given by (2), respectively by (3).

The following two sections will provide characterizations of the outcomes that an agenda setter can achieve under the amendment and successive procedure for a given dominance relation $P$.

\section{Choosing With the Amendment Procedure}

In this section we consider the case where society uses the amendment procedure for a given agenda to choose an alternative from $X$. Throughout we assume that the agenda incorporates all alternatives in $X$, i.e., for any agenda $\left(x_{1}, \ldots, x_{m}\right)$ it is true that $X=$ $\left\{x_{1}, \ldots, x_{m}\right\}$.

We assume that there is a dominance relation $P$ on $X$ and that the outcome of an agenda is determined according to (2). So as to characterize the set of alternatives that can be supported as the outcome for some agenda, we will assume that $P$ is complete or asymmetric. We first derive some auxiliary results. All proofs are provided in the Appendix.

For a given agenda $\left(x_{1}, \ldots, x_{m}\right)$ define the auxiliary alternatives $\bar{x}_{1}, \ldots, \bar{x}_{m}$ by

$$
\bar{x}_{k}=o^{A}\left(x_{k}, x_{k+1}, \ldots, x_{m}\right) \text { for } k=1, \ldots, m .
$$

Lemma 3.1. For all $k=1, \ldots, m-1$,

$$
\bar{x}_{k}=x_{k} \quad \Longleftrightarrow \quad x_{k} P \bar{x}_{l} \quad \text { for all } l=k+1, \ldots, m .
$$

\footnotetext{
${ }^{15}$ See Section 6 for a discussion of the alternative tie-breaking rule where $x_{1}$ is approved if and only if it is not defeated by $o^{S}\left(x_{2}, x_{3}, \ldots, x_{m}\right)$.
} 
Lemma 3.2. If $x_{k}=o^{A}\left(x_{1}, \ldots, x_{m}\right)$ for some $k$ with $1 \leq k \leq m$, then $x_{k}=\bar{x}_{k}$.

We are now ready to state our main characterization result that provides a necessary and sufficient condition for an alternative to be the outcome of some agenda under the amendment procedure.

TheOREM 3.1. Let $P$ be complete or asymmetric. Let $x \in X$ and let

$$
Y(x)=\{y \in X \mid y P x \text { and } \neg x P y\} .
$$

Then there exists an agenda $\left(x_{1}, \ldots, x_{m}\right)$ with $x=o^{A}\left(x_{1}, \ldots, x_{m}\right)$ if and only if for all $y \in Y(x)$, there is an alternative $z(y) \in X$, such that the following two conditions are satisfied.

(i) We have $\neg y P z(y)$ and $x P z(y)$.

(ii) There exists an ordering $\left(z_{1}, \ldots, z_{t}\right)$ of the alternatives in

$$
Z(x)=\{z \mid z=z(y) \text { for some } y \in Y(x)\},
$$

such that $z_{k} P z_{l}$ for all $k=1, \ldots, t-1$ and for all $l>k$.

Although the proof of the theorem is in the Appendix, let us briefly hint at the major ideas behind it. Regarding necessity, it is clear that the choice of $x$ is threatened by the existence of the elements of $Y(x)$, which would eliminate $x$ if ever really confronted against it. Hence, alternatives that are beaten by $x$ but not beaten by those in $Y(x)$ are needed, and these are the ones in the set $Z(x)$. The additional conditions on the dominance relation among the alternatives in $Z(x)$ are also needed to ensure that they can be presented in an appropriate order, so as to fulfill their role as deterrents of alternatives in $Y(x)$. The sufficiency part consists in exhibiting a way to order the alternatives that would deliver $x$ as an outcome, given that the conditions are satisfied. These orders depend on whether we consider the case of a complete or an asymmetric dominance relation. For the complete case, if $Y(x)$ is empty, then use any order where $x$ is the first alternative in the agenda. Otherwise, use the order

$$
\left(x, x_{1}, \ldots, x_{m-r-t-1}, y_{1}, \ldots, y_{r}, z_{1}, \ldots, z_{t}\right),
$$

where here the order of the $y_{i}$ 's is any, and the $x_{i}$ 's stand for those alternatives other than $x$ that do not belong to either $Y(x)$ or $Z(x)$. Similarly, for the asymmetric case, if $Y(x)$ is empty, use any order where $x$ is the last alternative in the agenda, and if $Y(x)$ is nonempty, use the order

$$
\left(x_{1}, \ldots, x_{m-r-t-1}, x, y_{1}, \ldots, y_{r}, z_{1}, \ldots, z_{t}\right),
$$

where again the order of the $y_{i}$ 's is any, and the $x_{i}$ 's stand for those alternatives other than $x$ that do not belong to either $Y(x)$ or $Z(x)$.

For later use we provide the following alternative characterization of the set of attainable alternatives under the amendment procedure. It is immediate to see that the following characterization is equivalent to the one in Theorem 3.1. 
Theorem 3.2. Let $P$ be complete or asymmetric. Let $x \in X$ and let

$$
Y(x)=\{y \in X \mid y P x \text { and } \neg x P y\} .
$$

Then there exists an agenda $\left(x_{1}, \ldots, x_{m}\right)$ with $x=o^{A}\left(x_{1}, \ldots, x_{m}\right)$ if and only if there is a set of alternatives $Z(x)$ with $x \notin Z(x)$ and $x P z$ for all $z \in Z(x)$, such that the following two conditions are satisfied:

(i) For all $y \in Y(x)$ there exists $a z \in Z(x)$ such that $\neg y P z$.

(ii) There exists an ordering $\left(z_{1}, \ldots, z_{t}\right)$ of the alternatives in $Z(x)$ such that $z_{k} P z_{l}$ for all $k=1, \ldots, t-1$ and for all $l>k$.

Clearly, in many cases one can attain a given alternative through several orders. Therefore, no uniqueness claim is placed on the orders that we use in the sufficiency part of the proof. However, it is interesting to realize that, in the complete case, placing in first place the alternative that one wants to obtain is always effective, in the following sense.

Corollary 3.1. Let $P$ be complete and let $\left(x_{1}, \ldots, x_{m}\right)$ be an agenda. If for some $k>1$, $x_{k}=o^{A}\left(x_{1}, \ldots, x_{m}\right)$, then there exists an agenda $\left(x_{1}^{\prime}, \ldots, x_{m}^{\prime}\right)$ with $x_{1}^{\prime}=x_{k}$ and

$$
x_{k}=o^{A}\left(x_{1}^{\prime}, \ldots, x_{m}^{\prime}\right) .
$$

The proof of Corollary 3.1 follows immediately from the constructive sufficiency proof of Theorem 3.1.

The following example shows that it is not sufficient to move the outcome of an agenda one or only a few steps forward. Unless it is moved to the first position in the agenda, it need not remain the outcome.

Example 3.1. Let $X=\left\{x_{1}, x_{2}, x_{3}\right\}$ and consider the dominance relation $P$ given by

$$
x_{2} P x_{1}, x_{2} P x_{3}, x_{3} P x_{1} \text {, and } x_{3} P x_{2} \cdot{ }^{16}
$$

Observe that $P$ is complete but not asymmetric. Then

$$
x_{3}=o^{A}\left(x_{1}, x_{2}, x_{3}\right)=o^{A}\left(x_{3}, x_{1}, x_{2}\right)=o^{A}\left(x_{3}, x_{2}, x_{1}\right)
$$

and

$$
x_{2}=o^{A}\left(x_{1}, x_{3}, x_{2}\right)=o^{A}\left(x_{2}, x_{3}, x_{1}\right) .
$$

For a given dominance relation $P$, an alternative $x$ belongs to the uncovered set (Miller 1980) if and only if for all $y \neq x$, either $x P y$ or there exists a $z \in X$ with $x P z$ and $z P y$. The following then is a straightforward corollary of Theorem 3.1.

\footnotetext{
${ }^{16}$ The following preference profile $\left(\mathcal{P}_{1}, \mathcal{P}_{2}, \mathcal{P}_{3}\right)$ for three voters generates $P$ for majority voting with quota $q=1: x_{3} \mathcal{P}_{i} x_{2} \mathcal{P}_{i} x_{1}$ for $i=1,2$ and $x_{2} \mathcal{P}_{3} x_{3} \mathcal{P}_{3} x_{1}$.
} 
Corollary 3.2. Let $P$ be complete and let $x=o^{A}\left(x_{1}, \ldots, x_{m}\right)$ for some agenda $\left(x_{1}, \ldots, x_{m}\right)$. Then $x$ belongs to the uncovered set.

Corollary 3.2 recovers Miller's (1980) result that in the case of a tournament, i.e., where $P$ is complete and asymmetric, the set of sophisticated voting outcomes for the amendment procedure is a subset of the uncovered set.

If $P$ is asymmetric, then Theorem 3.1 provides an alternative characterization of the set of possible outcomes to the one given in Banks and Bordes (1988, Theorem 3.6). To state their result, we need some additional definitions. For our tie-breaking rule, the pair $\left(X^{\prime}, d\right)$ with $X^{\prime} \subseteq X$ is a trajectory if $d: X^{\prime} \rightarrow\{1, \ldots, m\}$ is one-to-one and $d(x)>d(y)$ implies that $x P y$. A trajectory $\left(X^{\prime}, d\right)$ is maximal if for all $y \in X \backslash X^{\prime}$ the pair $\left(X^{\prime} \cup\{y\}, d^{\prime}\right)$ is not a trajectory, where $d^{\prime}(x)=d(x)$ for all $x \in X^{\prime}$ and $d(y)=\# X^{\prime}+1$.

Corollary 3.3 (Banks and Bordes 1988). If $P$ is asymmetric, then $x=o^{A}\left(x_{1}, \ldots, x_{m}\right)$ for some agenda $\left(x_{1}, \ldots, x_{m}\right)$ if and only if there exists a maximal trajectory $\left(X^{\prime}, d\right)$ with $d(x)=t$, where $t=\# X^{\prime}$.

\section{Choosing With the SUCCessive PRocedure}

We now turn to the case where society uses the successive procedure for a given agenda to choose an alternative from $X$. Hence, we assume that there is a dominance relation $P$ on $X$ and that the outcome of an agenda is determined according to (3). Again we first derive some auxiliary results before presenting the characterization of the set of alternatives that can be achieved as the outcome for some agenda. To this end, we define the auxiliary alternatives $\bar{x}_{k}$ by

$$
\bar{x}_{k}=o^{S}\left(x_{k}, x_{k+1}, \ldots, x_{m}\right) \text { for } k=1, \ldots, m .
$$

The first lemma shows that an alternative that was eliminated at some stage will never return.

Lemma 4.1. Let $\left(x_{1}, \ldots, x_{m}\right)$ be an agenda. If $\bar{x}_{k} \neq x_{s}$ for some $s \geq k$, then $\bar{x}_{l} \neq x_{s}$ for all $l<k$.

Lemma 4.1 immediately implies the following result.

LEMMA 4.2. We have $x_{k}=o^{S}\left(x_{1}, \ldots, x_{m}\right)$ for some $1 \leq k \leq m$ if and only if $\bar{x}_{l}=x_{k}$ for all $l \leq k$.

We are now ready to present our main characterization result for the successive procedure.

Theorem 4.1. Let $P$ be complete or asymmetric. Let $x \in X$ and let

$$
Y(x)=\{y \in X \mid y P x \text { and } \neg x P y\} .
$$

Then there exists an agenda $\left(x_{1}, \ldots, x_{m}\right)$ with $x=o^{S}\left(x_{1}, \ldots, x_{m}\right)$ if and only if there is a set of alternatives $Z(x)$ with $x \notin Z(x)$ such that the following two conditions are satisfied: 
(i) For all $y \in Y(x)$ there exists $a z \in Z(x)$ such that $z P$ if $P$ is complete and such that $\neg y P z$ if $P$ is asymmetric.

(ii) There exists an ordering $\left(z_{1}, \ldots, z_{t}\right)$ of the alternatives in $Z(x)$ such that $z_{l} P z_{l+1}$ for all $l=1, \ldots, t-1$ and $x P z_{1}$.

Again let us briefly dwell on the major ideas of the proof, which is given in the Appendix. For necessity, any alternative in $Y(x)$ that threatens $x$ must be eliminated before it meets $x$. This is achieved by the alternatives in $Z(x)$ that may in turn threaten $x$, but that can be placed in such an order that the alternative that actually meets $x$ is eliminated by $x$. For sufficiency, we must find an order of the alternatives that delivers $x$ as an outcome, given that the conditions are satisfied. This order again depends on whether we consider the case of a complete or an asymmetric dominance relation. For the complete case, if $Y(x)$ is empty, then $x P y$ for all $y \neq x$. Hence, $x$ beats the outcome of any agenda on the remaining alternatives, which implies that $x$ is the outcome of any agenda that has $x$ as the first alternative. Otherwise, if $Y(x) \neq \varnothing$, use the order

$$
\left(x, x_{1}, \ldots, x_{m-r-1}, w_{1}, \ldots, w_{r}\right) .
$$

Here, the $x_{i}$ 's are all alternatives other than $x$ that do not belong to either $Y(x)$ or $Z(x)$, and the $w_{i}$ 's are alternatives that belong either to $Y(x)$ or to $Z(x)$, and their order has to be selected in a delicate manner that is explained along the inductive proof. Similarly, for the asymmetric case, if $Y(x)$ is empty, then $\neg y P x$ for all $y \neq x$. Hence, no predecessor of $x$ in an agenda ever beats $x$ and we can use any order where $x$ is the last alternative to obtain $x$ as the outcome of the agenda. If $Y(x)$ is nonempty, use the order

$$
\left(x_{1}, \ldots, x_{m-r-1}, x, w_{1}, \ldots, w_{r}\right),
$$

where again the $x_{i}$ 's are all alternatives other than $x$ that do not belong to either $Y(x)$ or $Z(x)$ and the $w_{i}$ 's are alternatives that belong either to $Y(x)$ or to $Z(x)$ and that are ordered in a specific manner.

Like for the amendment procedure, if $P$ is complete and an alternative $x$ can be obtained as the outcome for some agenda, then $x$ is the outcome of some agenda where $x$ is placed first.

Corollary 4.1. Let $P$ be complete and let $\left(x_{1}, \ldots, x_{m}\right)$ be an agenda. If for some $k>1$, $x_{k}=o^{S}\left(x_{1}, \ldots, x_{m}\right)$, then there exists an agenda $\left(x_{1}^{\prime}, \ldots, x_{m}^{\prime}\right)$ with $x_{1}^{\prime}=x_{k}$ and

$$
x_{k}=o^{S}\left(x_{1}^{\prime}, \ldots, x_{m}^{\prime}\right) .
$$

The proof is again straightforward given the constructive sufficiency proof of Theorem 4.1 .

For the special case where $P$ is a tournament, i.e., complete and asymmetric, Theorem 4.1 recovers the well known result that the set of attainable outcomes under the successive procedure coincides with the top cycle (Miller 1977). We can even generalize this result to any complete and not necessarily asymmetric dominance relation $P$. To this end, we first define the top cycle for a complete dominance relation $P .{ }^{17}$ The top

\footnotetext{
${ }^{17}$ In Section 6 we will provide a definition of the top cycle for an asymmetric dominance relation.
} 
cycle of $P$ is the set of all alternatives $x$ such that for all $y \neq x$, there exists a sequence of alternatives $z_{0}, z_{1}, \ldots, z_{s}$, with $z_{0}=x, z_{s}=y$, and $z_{l} P z_{l+1}$ for all $l=1, \ldots, s-1$.

Corollary 4.2. If $P$ is complete, then $x=o^{S}\left(x_{1}, \ldots, x_{m}\right)$ for some agenda $\left(x_{1}, \ldots, x_{m}\right)$ if and only if $x$ is in the top cycle of $P$.

\section{ON THE FORMS AND EXTENT OF AGENDA MANIPULATION}

In this section we focus on the possibilities that an agenda setter may find to use her power to determine the order of vote in her own favor, so as to get a most preferred alternative. In its most demanding version, nonmanipulability would require that whoever is chosen as an agenda setter could not change the outcome at all, because it is the same regardless of the order of vote.

Definition 5.1. A sequential voting procedure is nonmanipulable by any agenda setter at a given dominance relation $P$ if it yields the same outcome regardless of the agenda.

Note that the definition applies to any potential agenda setter.

It turns out that both the amendment and the successive procedure are nonmanipulable whenever there exists a (generalized) Condorcet winner, i.e., an alternative that dominates all others and in turn is not dominated. Hence, both procedures are nonmanipulable on the same set of preference profiles. So as to state this result, for any dominance relation $P$ we let $O^{A}(P)\left(O^{S}(P)\right)$ denote the set of alternatives that are outcomes for some agenda under the amendment (successive) procedure given $P$.

Theorem 5.1. Let $P$ be complete or asymmetric and let $x \in X$. Then the following statements are equivalent:

(i) We have $O^{S}(P)=\{x\}$.

(ii) We have $O^{A}(P)=\{x\}$.

(iii) For all $y \in X$ with $y \neq x$ it is true that $x P y$ and $\neg y P x$.

For those profiles where several outcomes could be reached, depending on the order of vote, it is possible to compare the choice flexibility that an agenda setter may obtain from alternative rules, as expressed by the following definition.

Definition 5.2. Given two sequential voting procedures, we say that one is more agenda manipulable than the other if, for any complete or asymmetric dominance relation $P$, the set of alternatives that are attainable by agenda manipulation under the latter is a subset of the former, and it is a strict subset for at least one dominance relation $P$.

We can now state our first result on agenda manipulation. 
Proposition 5.1. The successive procedure is more agenda manipulable than the amendment procedure.

The claim that $O^{A}(P) \subseteq O^{S}(P)$ for all preference relations $P$ is an immediate implication of Theorems 3.2 and 4.1. To get an intuition for this result observe that the amendment procedure imposes stronger conditions on an alternative for it to survive the sequential voting procedure than the successive procedure. To obtain $x$ as the outcome of an agenda for the successive procedure, it is sufficient that $x$ dominates the outcome of some agenda for the remaining alternatives. Hence, it is sufficient to find some ordering $\left(x_{1}, \ldots, x_{m-1}\right)$ of the alternatives different from $x$, such that $x P o^{S}\left(x_{1}, \ldots, x_{m-1}\right)$ (see (3)). By contrast, for $x$ to be the outcome of the agenda $\left(x, x_{1}, \ldots, x_{m-1}\right)$ under the amendment procedure, $x$ must be the outcome of any agenda $\left(x, x_{k}, \ldots, x_{m-1}\right)$ for $k=1, \ldots, m-1$ (see (2)).

Observe that Proposition 5.1 generalizes a known result for tournaments to arbitrary preference relations or arbitrary quotas, respectively. ${ }^{18}$

To verify that there exist relations $P$ with $O^{A}(P) \varsubsetneqq O^{S}(P)$, consider the following example.

ExAmple 5.1. Let $X=\{x, y, w, z\}$ and let $P$ be given by

$$
x P w, y P x, y P w, w P z, z P x, \text { and } z P y{ }^{19}
$$

Then $x=o^{S}(x, w, z, y)$, but $x \notin O^{A}(P)$. In fact, only $y, w$, and $z$ satisfy conditions (i) and (ii) in Theorem 3.1.

In what follows we analyze the role of the quota in determining the degree of manipulability of our rules for the special case where the social relation $P$ is derived from a vote under a given quota. It turns out that the set of preference profiles at which the amendment and successive procedures are nonmanipulable is maximized at simple majority voting. To state this result, we denote by $\Phi(q)$ the set of profiles $\mathcal{P}$ such that there exists a generalized Condorcet winner under majority voting with quota $q$, i.e., $\Phi(q)$ is the set of profiles at which the amendment and the successive procedures are nonmanipulable given $q$ (cf. Theorem 5.1).

Proposition 5.2. Let $1 \leq q<q^{\prime} \leq\left\lfloor\frac{n}{2}\right\rfloor+1$ or $\left\lfloor\frac{n}{2}\right\rfloor+1 \leq q^{\prime}<q \leq n$. Then

$$
\Phi(q) \subseteq \Phi\left(q^{\prime}\right)
$$

In particular, $\Phi(q)$ is maximal for $q=\left\lfloor\frac{n}{2}\right\rfloor+1$, i.e., for simple majority voting. ${ }^{20}$

The intuition for this result is simple: By Theorem 5.1, the amendment and successive procedures are nonmanipulable at quota $q$ if there exists an alternative $x$ such that

\footnotetext{
${ }^{18}$ For tournaments, the result follows from the fact that the Banks set is a subset of the top cycle.

${ }^{19}$ The following preference profile $\left(\mathcal{P}_{1}, \mathcal{P}_{2}, \mathcal{P}_{3}\right)$ for three voters generates $P$ for simple majority voting: $y \mathcal{P}_{1} x \mathcal{P}_{1} w \mathcal{P}_{1} z, w \mathcal{P}_{2} z \mathcal{P}_{2} y \mathcal{P}_{2} x, z \mathcal{P}_{3} y \mathcal{P}_{3} x \mathcal{P}_{3} w$

${ }^{20}$ If $n$ is even, then $\Phi\left(\frac{n}{2}\right)=\Phi\left(\frac{n}{2}+1\right)$, i.e., the maximizer of $\Phi(q)$ is not unique.
} 
for all alternatives $y \neq x$, (i) $x$ is a $q$ winner over $y$ and (ii) $y$ is no $q$ winner over $x$. Now obviously (i) is easier to satisfy if $q$ is small while (ii) is easier to satisfy if $q$ is large. The simple majority quota balances the opposite effects that the quota has on the fulfillment of (i) and (ii). Hence, the domain of preference profiles at which the voting procedures are nonmanipulable is maximal for the simple majority quota.

We now fix a preference profile and compare the degree of manipulability across different quotas. Let $O^{A}(\mathcal{P}, q)\left(O^{S}(\mathcal{P}, q)\right)$ denote the set of alternatives that are outcomes under majority voting with quota $q$ at profile $\mathcal{P}$ for some agenda under the amendment (successive) procedure.

We first consider the amendment procedure. The following example shows that the sets $O^{A}(\mathcal{P}, q)$ are not nested in general.

ExAmple 5.2. Let $X=\{x, y, z\}$ and let there be five voters with preferences

$$
\begin{aligned}
& z \mathcal{P}_{i} y \mathcal{P}_{i} x \quad \text { for } i=1,2, \\
& y \mathcal{P}_{i} x \mathcal{P}_{i} z \quad \text { for } i=3,4, \\
& x \mathcal{P}_{5} z \mathcal{P}_{5} y
\end{aligned}
$$

Using Theorem 3.1 it is straightforward to verify that

$$
O^{A}(\mathcal{P}, 1)=O^{A}(\mathcal{P}, 3)=O^{A}(\mathcal{P}, 5)=\{x, y, z\} \quad \text { and } \quad O^{A}(\mathcal{P}, 2)=O^{A}(\mathcal{P}, 4)=\{y, z\} . \diamond
$$

While there is no quota that minimizes the degree of manipulability for the amendment procedure, unanimity turns out to be the one that maximizes it.

Proposition 5.3. For every preference profile $\mathcal{P}$ and for all $q=1, \ldots, n-1$, it is true that

$$
O^{A}(\mathcal{P}, q) \subseteq O^{A}(\mathcal{P}, n)
$$

The situation is somewhat different for the successive procedure. There, the sets $O^{S}(\mathcal{P}, q)$ are nested for submajority and simple majority quotas. Hence, simple majority is a manipulation minimizer among all submajority and simple majority quotas. However, nestedness does not hold for supermajority quotas. We summarize these results in the following proposition.

Proposition 5.4. Let $\mathcal{P}$ be an arbitrary preference profile. Then the following statements are true:

(i) For all $q, q^{\prime}$ with $1 \leq q<q^{\prime} \leq\left\lfloor\frac{n}{2}\right\rfloor+1$ it holds that $O^{S}\left(\mathcal{P}, q^{\prime}\right) \subseteq O^{S}(\mathcal{P}, q)$.

(ii) For all $q, q^{\prime}$ with $\left\lfloor\frac{n}{2}\right\rfloor+1 \leq q<q^{\prime} \leq n$ the sets $O^{S}(\mathcal{P}, q)$ and $O^{S}\left(\mathcal{P}, q^{\prime}\right)$ are not necessarily nested, i.e., there exist a set of alternatives $X$ and a preference profile $\mathcal{P}$ such that neither $O^{S}(\mathcal{P}, q) \subseteq O^{S}\left(\mathcal{P}, q^{\prime}\right)$ holds for all $q, q^{\prime}$ with $\left\lfloor\frac{n}{2}\right\rfloor+1 \leq q<q^{\prime} \leq n$ nor $O^{S}\left(\mathcal{P}, q^{\prime}\right) \subseteq O^{S}(\mathcal{P}, q)$ holds for all $q, q^{\prime}$ with $\left\lfloor\frac{n}{2}\right\rfloor+1 \leq q<q^{\prime} \leq n$. 
The first claim in Proposition 5.4 is proved in the Appendix. The intuition is as follows. For any submajority quota $q$ the social preference relation $P$ derived from majority voting with quota $q$ is complete. In this case conditions (i) and (ii) in Theorem 4.1 only involve dominance relations on pairs of alternatives that continue to hold for quotas smaller than $q$. Hence, if some alternative $x$ is attainable under some submajority quota $q^{\prime}$, it is also attainable under any quota $q<q^{\prime}$.

To prove the second claim in Proposition 5.4, observe that for the preferences in Example 5.2 we obtain

$$
O^{S}(\mathcal{P}, 1)=O^{S}(\mathcal{P}, 2)=O^{S}(\mathcal{P}, 3)=O^{S}(\mathcal{P}, 5)=\{x, y, z\}
$$

and

$$
O^{S}(\mathcal{P}, 4)=\{y, z\}
$$

The preference profile in Example 5.2 also demonstrates that it is not true that simple majority voting always minimizes the degree of manipulability, neither for the amendment nor for the successive procedure.

One source of the difference in the set of attainable outcomes under the amendment and successive procedures is that the former always selects an outcome in the Pareto set while the latter may also have inefficient outcomes as we will show below. For the special case of a tournament this is well known since in this case the attainable set for the amendment procedure is a subset of the uncovered set (see Corollary 3.2 and Miller 1980), which in turn is a subset of the Pareto set (see Miller 1980). Moreover, in case of a tournament the attainable set for the successive procedure coincides with the top cycle (see Corollary 4.2 and Miller 1977), which is not, in general, a subset of the Pareto set (see Example 6 in Miller 1995, and Theorem 10.2.3 in Laslier 1997). The following propositions extend to any arbitrary complete or asymmetric dominance relation what was known in the case of tournaments about the relationship between the Pareto set and the attainable sets for the amendment and successive procedure.

Proposition 5.5. (i) No Pareto dominated alternative is attainable under the amendment procedure for any $q=1, \ldots, n$, i.e.,

$$
O^{A}(\mathcal{P}, q) \subseteq\left\{x \mid \text { there exists no } y \text { with } y \mathcal{P}_{i} x \text { for all } i\right\} .
$$

(ii) For $q \in\{1, n\}$ the set of outcomes $O^{A}(\mathcal{P}, q)$ coincides with the set of alternatives that are not Pareto dominated, i.e.,

$$
O^{A}(\mathcal{P}, 1)=O^{A}(\mathcal{P}, n)=\left\{x \mid \text { there exists no } y \text { with } y \mathcal{P}_{i} x \text { for all } i\right\}
$$

For the successive procedure, $O^{S}(\mathcal{P}, n)$ is the set of alternatives that are not Pareto dominated by any other alternative. However, different from the amendment procedure, for the successive procedure and $q<n$ an alternative can be the outcome for some agenda even if it is Pareto dominated. In particular, it is not true in general that $O^{S}(\mathcal{P}, 1)=O^{S}(\mathcal{P}, n)$. 
Proposition 5.6. (i) We have $O^{S}(\mathcal{P}, n)=\left\{x \mid\right.$ there exists no y with $y \mathcal{P}_{i} x$ for all $\left.i\right\}$.

(ii) Let $n \geq 3$ and let $1 \leq q \leq n-1$. Then there exist a set of alternatives $X$ and voters' preferences $\mathcal{P}$ such that $x \in O^{S}(\mathcal{P}, q)$ for some Pareto dominated alternative $x \in X$.

The first claim in Proposition 5.6 is proved in the Appendix. The second claim is proved by the following examples.

Example 5.3. Let $X=\{x, y, z\}$ and let there be $n \geq 3$ voters with preferences

$$
\begin{aligned}
& z \mathcal{P}_{1} y \mathcal{P}_{1} x, \\
& y \mathcal{P}_{i} x \mathcal{P}_{i} z \text { for all } i=2, \ldots, n
\end{aligned}
$$

Then $x$ is Pareto dominated by $y$ and $x=o^{S}(x, z, y)$ for majority voting with quota $q=1$, i.e., $x \in O^{S}(\mathcal{P}, 1)$.

Example 5.4. Let $n \geq 3$ and $2 \leq q \leq n-1$. Let $X=\{x, y, w, z\}$ and let there be $n$ voters with preferences

$$
\begin{aligned}
& w \mathcal{P}_{1} z \mathcal{P}_{1} y \mathcal{P}_{1} x, \\
& z \mathcal{P}_{2} y \mathcal{P}_{2} x \mathcal{P}_{2} w, \\
& y \mathcal{P}_{i} x \mathcal{P}_{i} w \mathcal{P}_{i} z, \quad \text { for all } i=3, \ldots, q+1
\end{aligned}
$$

If $q<n-1$, let

$$
z \mathcal{P}_{i} y \mathcal{P}_{i} w \mathcal{P}_{i} x, \quad \text { for all } i=q+2, \ldots, n .
$$

Then $x$ is Pareto dominated by $y$ and $x=o^{S}(x, w, y, z)$ for majority voting with quota $q$, i.e., $x \in O^{S}(\mathcal{P}, q)$.

As shown by Example 5.2, the difference in the Pareto properties is not the only source of a difference between $O^{A}(\mathcal{P}, q)$ and $O^{S}(\mathcal{P}, q)$. In this example, $x \in O^{S}(\mathcal{P}, 2)$ and $x \notin O^{A}(\mathcal{P}, 2)$, but $x$ is not Pareto dominated.

\section{Robustness}

In Section 2 we have already pointed to the role of the convention whereby, in defining our rules, we attribute to alternatives in one of the directions, right or left in the tree, the opportunity to stay for the next stage in the vote. We can think of that convention as assigning either the left or right successor $(l(\nu)$ or $r(\nu))$ the role of a temporary status quo, and assuming that it will prevail if and only if it is not beaten by the other successor node in a $q$-majority vote. Unless there are constraints on the formation of an agenda, as in legislative voting, it seems arbitrary whether one treats the left or right successor node as 
the temporary status quo. ${ }^{21}$ In fact, as Banks and Bordes (1988, p. 36) state when considering the amendment procedure, "two 'natural' tie-breaking rules are (i) the alternative introduced earlier proceeds to the next vote [...] and (ii) the alternative introduced later continues." Up to this point we have considered the second rule, which we will call the backward looking rule because it treats the right successor, i.e., later (higher numbered) alternatives, as a temporary status quo. In the following discussion we will show that our main results continue to hold under the first rule, which we will call the forward looking rule, because it treats the left successor, i.e., earlier (lower numbered) alternatives, as a temporary status quo. We will also point to some interesting modifications that obtain under the forward looking rule. We do not provide any proofs for the results in this section but instead refer the reader to Barberà and Gerber (2015) where the proofs can be found.

Using the forward looking rule we obtain the following modifications in the inductive definition of the outcome of an agenda. For the amendment procedure replace (2) by

$$
\begin{aligned}
& o^{A}\left(x_{1}, x_{2}, \ldots, x_{m}\right) \\
& \quad= \begin{cases}o^{A}\left(x_{1}, x_{3}, \ldots, x_{m}\right) & \text { if } \neg o^{A}\left(x_{2}, x_{3}, \ldots, x_{m}\right) P o^{A}\left(x_{1}, x_{3}, \ldots, x_{m}\right), \\
o^{A}\left(x_{2}, x_{3}, \ldots, x_{m}\right) & \text { if } o^{A}\left(x_{2}, x_{3}, \ldots, x_{m}\right) P o^{A}\left(x_{1}, x_{3}, \ldots, x_{m}\right) .\end{cases}
\end{aligned}
$$

Similarly, for the successive procedure replace (3) by

$$
o^{S}\left(x_{1}, x_{2}, \ldots, x_{m}\right)= \begin{cases}x_{1} & \text { if } \neg o^{S}\left(x_{2}, x_{3}, \ldots, x_{m}\right) P x_{1} \\ o_{\left(x_{2}, x_{3}, \ldots, x_{m}\right)} & \text { if } o_{\left(x_{2}, x_{3}, \ldots, x_{m}\right) P x_{1}}\end{cases}
$$

With these definitions the attainable alternatives under the amendment and successive procedure can be characterized as follows.

Theorem 6.1. Let $P$ be complete or asymmetric. Let $x \in X$ and let

$$
Y(x)=\{y \in X \mid y P x \text { and } \neg x P y\} .
$$

Then there exists an agenda $\left(x_{1}, \ldots, x_{m}\right)$ with $x=o^{A}\left(x_{1}, \ldots, x_{m}\right)$ if and only if there is a set of alternatives $Z(x)$ with $x \notin Z(x)$ and $\neg z P x$ for all $z \in Z(x)$, such that the following two conditions are satisfied.

(i) For all $y \in Y(x)$ there exists $a z \in Z(x)$ such that $z P y$.

(ii) There exists an ordering $\left(z_{1}, \ldots, z_{t}\right)$ of the alternatives in $Z(x)$ such that $\neg z_{l} P z_{k}$ for all $k=1, \ldots, t-1$ and for all $l>k$.

\footnotetext{
${ }^{21}$ In legislative voting there is the convention to build the agenda $\left(x_{1}, \ldots, x_{m}\right)$ backward (Shepsle and Weingast 1984), i.e., $x_{m}$ is the status quo bill, $x_{m-1}$ is an alternative bill, $x_{m-2}$ is the alternative bill with an amendment, and so on. In this case, the natural convention is to give priority to alternatives that come later in the agenda, which is the rule we used so far.
} 
Theorem 6.2. Let $P$ be complete or asymmetric. Let $x \in X$ and let

$$
Y(x)=\{y \in X \mid y P x \text { and } \neg x P y\} .
$$

Then there exists an agenda $\left(x_{1}, \ldots, x_{m}\right)$ with $x=o^{S}\left(x_{1}, \ldots, x_{m}\right)$ if and only if there is a set of alternatives $Z(x)$ with $x \notin Z(x)$ such that the following two conditions are satisfied:

(i) For all $y \in Y(x)$ there exists $a z \in Z(x)$ such that $z P$ y if $P$ is complete and such that $\neg y P z$ if $P$ is asymmetric.

(ii) There exists an ordering $\left(z_{1}, \ldots, z_{t}\right)$ of the alternatives in $Z(x)$ such that $\neg z_{l+1} P z_{l}$ for all $l=1, \ldots, t-1$, and $\neg z_{1} P x$.

It is then immediate to see that Proposition 5.1 continues to hold under the forward looking rule. ${ }^{22}$ The same is true for our results concerning the nonmanipulability of the voting procedures (Theorem 5.1) and the domain of preference profiles for which the procedures are nonmanipulable under a given quota (Proposition 5.2). Moreover, all additional results for the amendment procedure in Section 5 concerning the nonnestedness of the attainable sets and the efficiency properties continue to hold under the forward looking rule.

Let us now come to some interesting modifications of our previous results under the forward looking rule. All modifications pertain to the successive procedure. The first concerns the relation between the top cycle and the set of outcomes under the successive procedure. While under the backward looking rule we showed that the two sets coincide if $P$ is complete (Corollary 4.2), under the forward looking rule the two sets coincide if $P$ is asymmetric. To state this result, we first have to define the top cycle for an asymmetric dominance relation $P$. To this end, let $R$ be the binary relation on $X$ defined by $x R y$ if and only if $\neg y P x$ for $x, y \in X$. Observe that $R$ is complete since $P$ is asymmetric. The top cycle of $P$ then is the set of all alternatives $x$ such that for all $y \neq x$, there exists a sequence of alternatives $z_{0}, z_{1}, \ldots, z_{s}$, with $z_{0}=x, z_{s}=y$, and $z_{l} R z_{l+1}$ for all $l=1, \ldots, s-1$. We then get the following corollary to Theorem 6.2.

CoRollary 6.1. If $P$ is asymmetric, then $x=o^{S}\left(x_{1}, \ldots, x_{m}\right)$ for some agenda $\left(x_{1}, \ldots, x_{m}\right)$ if and only if $x$ is in the top cycle of $P$.

Moreover, under the forward looking rule the sets of attainable alternatives under the successive procedure are nested for supermajority but not for submajority quotas, i.e., we have the following modification of Proposition 5.4. ${ }^{23}$

Proposition 6.1. Let $\mathcal{P}$ be an arbitrary preference profile. Then the following statements are true:

\footnotetext{
${ }^{22}$ Note that Example 5.1 continues to hold under the forward looking rule since the dominance relation is complete and asymmetric so that no ties ever obtain.

${ }^{23}$ The first claim in Proposition 6.1 can again be proved with the preference profile in Example 5.2 for which we obtain $O^{S}(\mathcal{P}, 1)=O^{S}(\mathcal{P}, 3)=O^{S}(\mathcal{P}, 4)=O^{S}(\mathcal{P}, 5)=\{x, y, z\}$ and $O^{S}(\mathcal{P}, 2)=\{y, z\}$ under the forward looking rule.
} 
(i) For all $q, q^{\prime}$, with $1 \leq q<q^{\prime} \leq\left\lfloor\frac{n}{2}\right\rfloor+1$ the sets $O^{S}(\mathcal{P}, q)$ and $O^{S}\left(\mathcal{P}, q^{\prime}\right)$ are not necessarily nested, i.e., there exist a set of alternatives $X$ and a preference profile $\mathcal{P}$ such that neither $O^{S}(\mathcal{P}, q) \subseteq O^{S}\left(\mathcal{P}, q^{\prime}\right)$ holds for all $q, q^{\prime}$ with $1 \leq q<q^{\prime} \leq\left\lfloor\frac{n}{2}\right\rfloor+1$ nor $O^{S}\left(\mathcal{P}, q^{\prime}\right) \subseteq O^{S}(\mathcal{P}, q)$ holds for all $q, q^{\prime}$ with $1 \leq q<q^{\prime} \leq\left\lfloor\frac{n}{2}\right\rfloor+1$.

(ii) For all $q, q^{\prime}$, with $\left\lfloor\frac{n}{2}\right\rfloor+1 \leq q<q^{\prime} \leq n$ it holds that $O^{S}(\mathcal{P}, q) \subseteq O^{S}\left(\mathcal{P}, q^{\prime}\right)$.

Finally, under the forward looking rule the set of attainable alternatives for the successive procedure coincides with the set of undominated alternatives for quota 1 but not for quota $n$. In fact, for all quotas different from 1 the successive procedure may select a dominated alternative under the forward looking rule. This is true, in particular, for the unanimity rule. To see this, consider the following example.

Example 6.1. Let $X=\{x, y, z\}$ and let there be three voters with preferences

$$
\begin{aligned}
& z \mathcal{P}_{i} y \mathcal{P}_{i} x \quad \text { for } i=1,2, \\
& y \mathcal{P}_{3} x \mathcal{P}_{3} z
\end{aligned}
$$

Then $x$ is Pareto dominated by $y$, but nevertheless $x=o^{S}(x, z, y)$ for majority voting with quota $q=3$.

\section{Conclusion}

It is well known that sequential voting procedures are prone to agenda manipulation except for very special cases, where there is a unique alternative that is the outcome under every agenda at a given profile of voters' preferences. Nevertheless, to the best of our knowledge our paper is the first to provide a comprehensive analysis of whether and how the voting procedures derived from the amendment and successive procedures with different majority quotas differ with respect to the scope of manipulation they permit.

Our analysis builds upon a characterization of the attainable sets for the amendment and successive procedures for arbitrary majority quotas. Using this characterization we can show that a well known result for tournaments extends to arbitrary majority quotas, namely that the successive procedure is uniformly more vulnerable toward agenda manipulation than the amendment procedure. This gives support to using the amendment rather than the successive procedure if the possibility of agenda manipulation is a concern in a committee or, more generally, in any democratic institution. We have also shown that the set of preference profiles for which neither procedure is manipulable is maximal under simple majority voting. However, when manipulation is possible, the connection between the degree of manipulability and the choice of a quota is complex. In particular, simple majority need no longer be the quota that minimizes the size of choices available to the agenda setter. 


\section{Appendix}

Proof of Lemma 3.1. The proof is by induction over $m$. For $m=2$ the claim immediately follows from (2). So assume that the claim has been proved for all agendas with at most $m-1$ alternatives and consider an agenda with $m$ alternatives, $\left(x_{1}, \ldots, x_{m}\right)$. By assumption, the claim holds for all $k=2, \ldots, m$, and it remains to consider $k=1$.

To prove necessity assume that $\bar{x}_{1}=x_{1}$. By (2), $\bar{x}_{1}=x_{1}$ implies that $x_{1}=o^{A}\left(x_{1}, x_{3}\right.$, $\left.\ldots, x_{m}\right)$. Since there are $m-1$ alternatives in the agenda $\left(x_{1}, x_{3}, \ldots, x_{m}\right)$, it follows that $x_{1} P \bar{x}_{k}$ for all $k=3, \ldots, m$ (observe that the auxiliary variables for agenda $\left(x_{1}, x_{3}, \ldots, x_{m}\right)$ defined in (4) are identical to those for agenda $\left(x_{1}, \ldots, x_{m}\right)$ whenever $\left.k \geq 3\right)$. Moreover, by (2), $o^{A}\left(x_{1}, \ldots, x_{m}\right)=o^{A}\left(x_{1}, x_{3}, \ldots, x_{m}\right)=x_{1}$ if and only if $x_{1} P o^{A}\left(x_{2}, \ldots, x_{m}\right)$, where the latter is equivalent to $x_{1} P \bar{x}_{2}$.

For sufficiency assume that $x_{1} P \bar{x}_{l}$ for all $l=2, \ldots, m$. Then, by (2), $o^{A}\left(x_{1}, \ldots, x_{m}\right) \neq$ $x_{1}$ implies that either $x_{1} \neq o^{A}\left(x_{1}, x_{3}, \ldots, x_{m}\right)$ or $x_{1}=o^{A}\left(x_{1}, x_{3}, \ldots, x_{m}\right)$ and $\neg x_{1} P$ $o^{A}\left(x_{2}, \ldots, x_{m}\right)$, which holds if and only if $\neg x_{1} P \bar{x}_{2}$. The latter case immediately leads to a contradiction since we have assumed that $x_{1} P \bar{x}_{2}$. It remains to consider the case where $x_{1} \neq o^{A}\left(x_{1}, x_{3}, \ldots, x_{m}\right)$. Because the agenda $\left(x_{1}, x_{3}, \ldots, x_{m}\right)$ has $m-1$ alternatives, we conclude that there must exist a $k \geq 3$ with $\neg x_{1} P \bar{x}_{k}$, which contradicts our assumption that $x_{1} P \bar{x}_{l}$ for all $l=2, \ldots, m$. This proves the claim.

Proof of Lemma 3.2. If $k=1$, nothing has to be shown. Hence, assume that $k \geq 2$. The proof is by induction over $m$. For $m=2$ nothing has to be proved if $k=2$. Hence, assume that the claim is true for any agenda with up to $m \geq 2$ alternatives and consider an agenda with $m+1$ alternatives. Let $x_{k}=o^{A}\left(x_{1}, \ldots, x_{m+1}\right)$. By definition of the outcome of an agenda this implies that

$$
x_{k} \in\left\{o^{A}\left(x_{1}, x_{3}, \ldots, x_{m+1}\right), o^{A}\left(x_{2}, \ldots, x_{m+1}\right)\right\} .
$$

Since both agendas $\left(x_{1}, x_{3}, \ldots, x_{m+1}\right)$ and $\left(x_{2}, \ldots, x_{m+1}\right)$ have $m$ alternatives, it follows in either case that $x_{k}=o^{A}\left(x_{k}, \ldots, x_{m+1}\right)$.

\section{Proof of Theorem 3.1.}

Necessity. Let $x_{k}=o^{A}\left(x_{1}, \ldots, x_{m}\right)$ for some $k \in\{1, \ldots, m\}$. From Lemma 3.2 it follows that $\bar{x}_{k}=x_{k}$ and then Lemma 3.1 implies that $x_{k} P \bar{x}_{l}$ for all $l>k$. We will now show that for all $l<k, x_{k}=\bar{x}_{l}$ or $x_{k} P \bar{x}_{l}$. Suppose by way of contradiction that there exists $l<k$ with $x_{k} \neq \bar{x}_{l}$ and $\neg x_{k} P \bar{x}_{l}$. We will prove that this implies, that $\bar{x}_{s} \neq x_{k}$ for all $s=1, \ldots, l-1$, where the case $s=1$ yields a contradiction to the assumption that $x_{k}=\bar{x}_{1}=o^{A}\left(x_{1}, \ldots, x_{m}\right)$.

The proof is by backward induction over $s$. Let $s=l-1$ and suppose by way of contradiction that $\bar{x}_{l-1}=x_{k}$. Since

$$
\bar{x}_{l-1} \in\left\{o^{A}\left(x_{l-1}, x_{l+1}, \ldots, x_{m}\right), \bar{x}_{l}\right\},
$$

this implies $x_{k}=\bar{x}_{l-1}=o^{A}\left(x_{l-1}, x_{l+1}, \ldots, x_{m}\right)$ and $x_{k} P \bar{x}_{l}$, which is a contradiction. Hence, $\bar{x}_{l-1} \neq x_{k}$. Assume we have shown that $\bar{x}_{s} \neq x_{k}$ for all $s$ with $t \leq s \leq l-1$, where 
$2 \leq t \leq l-1$. Suppose by way of contradiction that $\bar{x}_{t-1}=x_{k}$. Since

$$
\bar{x}_{t-1} \in\left\{o^{A}\left(x_{t-1}, x_{t+1}, \ldots, x_{m}\right), \bar{x}_{t}\right\},
$$

this implies $x_{k}=\bar{x}_{t-1}=o^{A}\left(x_{t-1}, x_{t+1}, \ldots, x_{m}\right)$. Since

$$
o^{A}\left(x_{t-1}, x_{t+1}, \ldots, x_{m}\right) \in\left\{o^{A}\left(x_{t-1}, x_{t+2}, \ldots, x_{m}\right), \bar{x}_{t+1}\right\}
$$

and $\bar{x}_{t+1} \neq x_{k}$, it follows that $x_{k}=o^{A}\left(x_{t-1}, x_{t+2}, \ldots, x_{m}\right)$. Continuing in this manner we conclude that

$$
x_{k} \in\left\{o^{A}\left(x_{t-1}, x_{l+1}, \ldots, x_{m}\right), \bar{x}_{l}\right\}
$$

and hence $x_{k}=o^{A}\left(x_{t-1}, x_{l+1}, \ldots, x_{m}\right)$, which is impossible given that $\neg x_{k} P \bar{x}_{l}$.

Summarizing, we have shown that for all $l \neq k, x_{k}=\bar{x}_{l}$ or $x_{k} P \bar{x}_{l}$. Returning to the proof of necessity we first note that nothing has to be proved if $Y\left(x_{k}\right)=\varnothing$. Hence, let $Y\left(x_{k}\right) \neq \varnothing$ and let $x_{l} \in Y\left(x_{k}\right)$, i.e., $x_{l} P x_{k}$ and $\neg x_{k} P x_{l}$. Then by our previous argument $\bar{x}_{l} \neq x_{l}$. Hence, from Lemma 3.1 it follows that there exists $l^{\prime}>l$ with $\neg x_{l} P \bar{x}_{l^{\prime}}$. By what we have shown above $x_{k} P \bar{x}_{l^{\prime}}$. Moreover, either $x_{l^{\prime}}=\bar{x}_{l^{\prime}}$ and $x_{k} P x_{l^{\prime}}$ or there exists $l^{\prime \prime}>l^{\prime}$ with $\bar{x}_{l^{\prime \prime}}=x_{l^{\prime \prime}}=\bar{x}_{l^{\prime}}$. Also in this case $x_{k} P x_{l^{\prime \prime}}$. This proves that for all $y \in Y\left(x_{k}\right)$ there exists $z(y) \in X$ with $\overline{z(y)}=z(y), \neg y P z(y)$ and $x_{k} P z(y)$, i.e., in particular (i) holds. Let $Z\left(x_{k}\right)=$ $\left\{z \mid z=z(y)\right.$ for some $\left.y \in Y\left(x_{k}\right)\right\}$ and let $\left(z_{1}, \ldots, z_{t}\right)$ be the ordering of the alternatives in $Z\left(x_{k}\right)$ in the agenda of which $x_{k}$ is the outcome. Since we have shown that $\bar{z}_{s}=z_{s}$ for all $s=1, \ldots, t$, Lemma 3.1 implies that (ii) holds.

Sufficiency. The proof is by construction. We consider the cases where $P$ is complete and where $P$ is asymmetric.

Case 1: $P$ is complete. Let $x$ be an alternative such that for all $y \in Y(x)$ there exists an alternative $z(y) \in X$ such that conditions (i) and (ii) are satisfied. If $Y(x)=\varnothing$, then by completeness of $P, x P$ for all alternatives $y \neq x$ and hence $x$ is the outcome of any agenda $\left(x_{1}, \ldots, x_{m}\right)$ with $x_{1}=x$. If $Y(x) \neq \varnothing$, let $\left(z_{1}, \ldots, z_{t}\right)$ be the ordering of the alternatives in $Z(x)$ with the property as given in (ii). Observe that $z_{k} \neq x$ for all $k=1, \ldots, t$, since $y P x$ and $\neg y P z_{k}$ for all $y \in Y(x)$. Take an arbitrary order $\left(y_{1}, \ldots, y_{r}\right)$ of the alternatives in $Y(x)$. If $r+t+1<m$, let $\left(x_{1}, \ldots, x_{m-r-t-1}\right)$ be an arbitrary order of the set of alternatives in $X \backslash(Y(x) \cup Z(x) \cup\{x\}) \neq \varnothing$. Consider the agenda $\left(x, x_{1}, \ldots, x_{m-r-t-1}, y_{1}, \ldots, y_{r}, z_{1}, \ldots, z_{t}\right)$ (if $r+t+1=m$, the agenda is $\left.\left(x, y_{1}, \ldots, y_{r}, z_{1}, \ldots, z_{t}\right)\right)$. We will now verify that

$$
x=o^{A}\left(x, x_{1}, \ldots, x_{m-r-t-1}, y_{1}, \ldots, y_{r}, z_{1}, \ldots, z_{t}\right) .
$$

By Lemma 3.1 it is sufficient to show that

(a) $x P o^{A}\left(z_{k}, \ldots, z_{t}\right)$ for all $k=1, \ldots, t$.

(b) $x P o^{A}\left(y_{k}, \ldots, y_{r}, z_{1}, \ldots, z_{t}\right)$ for all $k=1, \ldots, r$.

(c) $x \mathrm{Po}^{A}\left(x_{k}, \ldots, x_{m-r-t-1}, y_{1}, \ldots, y_{r}, z_{1}, \ldots, z_{t}\right)$ for all $k=1, \ldots, m-r-t-1$. 
Statement (a) follows from the fact that $o^{A}\left(z_{k}, \ldots, z_{t}\right)=z_{k}$ by Lemma 3.1 and $x P z_{k}$ for all $k=1, \ldots, t$. Statements (b) will follow from the fact that $o^{A}\left(y_{k}, \ldots, y_{r}, z_{1}, \ldots, z_{t}\right) \in$ $\left\{z_{1}, \ldots, z_{t}\right\}$ for all $k=1, \ldots, r$ and $x P z_{s}$ for all $s=1, \ldots, t$. We prove the latter by showing that

$$
o^{A}\left(y_{1}^{\prime}, \ldots, y_{l}^{\prime}, z_{1}, \ldots, z_{t}\right) \in\left\{z_{1}, \ldots, z_{t}\right\}
$$

for any agenda with $y_{1}^{\prime}, \ldots, y_{l}^{\prime} \in Y(x)$ and $l=1, \ldots, r$. The proof is by induction over $l$. Let $l=1$. Then by Lemma 3.1 and the definition of $Z(x), o^{A}\left(y_{1}^{\prime}, z_{1}, \ldots, z_{t}\right) \in\left\{z_{1}, \ldots, z_{t}\right\}$. Suppose (5) has been shown for all subsets of $Y(x)$ with at most $l \geq 1$ alternatives, where $l \leq r-1$. Consider now the agenda $\left(y_{1}^{\prime}, \ldots, y_{l+1}^{\prime}, z_{1}, \ldots, z_{t}\right)$ with $l+1$ alternatives from $Y(x)$. By definition of the outcome of an agenda,

$$
\begin{aligned}
& o^{A}\left(y_{1}^{\prime}, \ldots, y_{l+1}^{\prime}, z_{1}, \ldots, z_{t}\right) \\
& \quad \in\left\{o^{A}\left(y_{1}^{\prime}, y_{3}^{\prime}, \ldots, y_{l+1}^{\prime}, z_{1}, \ldots, z_{t}\right), o^{A}\left(y_{2}^{\prime}, \ldots, y_{l+1}^{\prime}, z_{1}, \ldots, z_{t}\right)\right\} .
\end{aligned}
$$

Since there are $l$ alternatives from $Y(x)$ in the agendas $\left(y_{1}^{\prime}, y_{3}^{\prime}, \ldots, y_{l+1}^{\prime}, z_{1}, \ldots, z_{t}\right)$ and $\left(y_{2}^{\prime}, \ldots, y_{l+1}^{\prime}, z_{1}, \ldots, z_{t}\right)$, it follows that the outcome of these agendas is an alternative in $\left\{z_{1}, \ldots, z_{t}\right\}$, which implies that $o^{A}\left(y_{1}^{\prime}, \ldots, y_{l+1}^{\prime}, z_{1}, \ldots, z_{t}\right) \in\left\{z_{1}, \ldots, z_{t}\right\}$. This proves (b).

To prove (c), suppose by way of contradiction that

$$
\neg x P o^{A}\left(x_{k}, \ldots, x_{m-r-t-1}, y_{1}, \ldots, y_{r}, z_{1}, \ldots, z_{t}\right)
$$

for some $k \in\{1, \ldots, m-r-t-1\}$. Since $x P x_{l}$ for all $l=1, \ldots, m-r-t-1$ and $x P z_{s}$ for all $s=1, \ldots, t$, this implies that

$$
o^{A}\left(x_{k}, \ldots, x_{m-r-t-1}, y_{1}, \ldots, y_{r}, z_{1}, \ldots, z_{t}\right) \in\left\{y_{1}, \ldots, y_{r}\right\} .
$$

Using Lemma 3.2 we conclude that there exists $k \in\{1, \ldots, r\}$ such that $y_{k}=o^{A}\left(y_{k}, \ldots, y_{r}\right.$, $\left.z_{1}, \ldots, z_{t}\right)$. However, this contradicts (5) and hence (c) holds as claimed.

Case 2: $P$ is asymmetric. Let $x$ be an alternative such that for all $y \in Y(x)$ there exists an alternative $z(y) \in X$ such that conditions (i) and (ii) are satisfied. If $Y(x)=\varnothing$, then from Lemmas 3.1 and 3.2 it follows that $x$ is the outcome of any agenda $\left(x_{1}, \ldots, x_{m}\right)$ with $x_{m}=x$. If $Y(x) \neq \varnothing$, let $\left(y_{1}, \ldots, y_{r}\right)$ be an arbitrary ordering of the alternatives in $Y(x)$. Moreover, let $\left(z_{1}, \ldots, z_{t}\right)$ be the ordering of the alternatives in $Z(x)$ with the property as given in (ii). As in Case 1 observe that $z_{k} \neq x$ for all $k=1, \ldots, t$. If $r+t+1<m$, let $\left(x_{1}, \ldots, x_{m-r-t-1}\right)$ be an arbitrary ordering of the set of alternatives in $X \backslash(Y(x) \cup Z(x) \cup$ $\{x\})$. Consider the agenda $\left(x_{1}, \ldots, x_{m-r-t-1}, x, y_{1}, \ldots, y_{r}, z_{1}, \ldots, z_{t}\right)$ (if $r+t+1=m$, the agenda is $\left.\left(x, y_{1}, \ldots, y_{r}, z_{1}, \ldots, z_{t}\right)\right)$. We will now verify that

$$
x=o^{A}\left(x_{1}, \ldots, x_{m-r-t-1}, x, y_{1}, \ldots, y_{r}, z_{1}, \ldots, z_{t}\right) .
$$

As in the case where $P$ is complete, we can show that $o^{A}\left(z_{k}, \ldots, z_{t}\right)=z_{k}$ for all $k=$ $1, \ldots, t$, and $o^{A}\left(y_{k}, \ldots, y_{r}, z_{1}, \ldots, z_{t}\right) \in\left\{z_{1}, \ldots, z_{t}\right\}$ for all $k=1, \ldots, r$. Since $x P z_{s}$ for all $s=1, \ldots, t$, Lemma 3.1 implies that $o^{A}\left(x, y_{1}, \ldots, y_{r}, z_{1}, \ldots, z_{t}\right)=x$. To complete the proof we show that

$$
o^{A}\left(x_{1}^{\prime}, \ldots, x_{l}^{\prime}, x, y_{1}, \ldots, y_{r}, z_{1}, \ldots, z_{t}\right)=x
$$


for any agenda with $x_{1}^{\prime}, \ldots, x_{l}^{\prime} \in X \backslash(Y(x) \cup Z(x) \cup\{x\})$ and $l=1, \ldots, m-r-t-1$. The proof is by induction over $l$. Let $l=1$. Suppose by way of contradiction that $o^{A}\left(x_{1}^{\prime}, x, y_{1}, \ldots, y_{r}, z_{1}, \ldots, z_{t}\right) \neq x$. Since

$$
\begin{aligned}
o^{A}\left(x_{1}^{\prime}, x, y_{1}, \ldots, y_{r}, z_{1}, \ldots, z_{t}\right) & \\
& \in\left\{o^{A}\left(x_{1}^{\prime}, y_{1}, \ldots, y_{r}, z_{1}, \ldots, z_{t}\right), o^{A}\left(x, y_{1}, \ldots, y_{r}, z_{1}, \ldots, z_{t}\right)\right\}
\end{aligned}
$$

and $o^{A}\left(x, y_{1}, \ldots, y_{r}, z_{1}, \ldots, z_{t}\right)=x$, it follows that $o^{A}\left(x_{1}^{\prime}, x, y_{1}, \ldots, y_{r}, z_{1}, \ldots, z_{t}\right)=$ $o^{A}\left(x_{1}^{\prime}, y_{1}, \ldots, y_{r}, z_{1}, \ldots, z_{t}\right)$ and $o^{A}\left(x_{1}^{\prime}, y_{1}, \ldots, y_{r}, z_{1}, \ldots, z_{t}\right) P x$. This implies that $o^{A}\left(x_{1}^{\prime}, y_{1}, \ldots, y_{r}, z_{1}, \ldots, z_{t}\right) \in\left\{y_{1}, \ldots, y_{r}\right\}$ since $\neg x_{1}^{\prime} P x$ and that $\neg z_{s} P x$ for all $s=1, \ldots, t$, since $P$ is asymmetric and $x P z_{s}$ for all $s=1, \ldots, t$. However, $o^{A}\left(x_{1}^{\prime}, y_{1}, \ldots, y_{r}, z_{1}, \ldots\right.$, $\left.z_{t}\right)=y_{k}$ for some $k \in\{1, \ldots, r\}$ implies that $y_{k}=o^{A}\left(y_{k}, \ldots, y_{r}, z_{1}, \ldots, z_{t}\right)$ by Lemma 3.2, which is a contradiction to what we have shown above. Hence, (6) holds for $l=1$.

Suppose (6) has been shown for all for any agenda with $x_{1}^{\prime}, \ldots, x_{l}^{\prime} \in X \backslash(Y(x) \cup Z(x) \cup$ $\{x\})$ with at most $l \geq 1$ alternatives, where $l \leq m-r-t-1$. Consider now the agenda $\left(x_{1}^{\prime}, \ldots, x_{l+1}^{\prime}, x, y_{1}, \ldots, y_{r}, z_{1}, \ldots, z_{t}\right)$ with $x_{1}^{\prime}, \ldots, x_{l+1}^{\prime} \in X \backslash(Y(x) \cup Z(x) \cup\{x\})$. Then

$$
\begin{aligned}
o^{A}\left(x_{1}^{\prime}, \ldots, x_{l+1}^{\prime}, x, y_{1}, \ldots, y_{r}, z_{1}, \ldots, z_{t}\right) & \\
& \in\left\{o^{A}\left(x_{1}^{\prime}, x_{3}^{\prime}, \ldots, x_{l+1}^{\prime}, x, y_{1}, \ldots, y_{r}, z_{1}, \ldots, z_{t}\right),\right. \\
& \left.o^{A}\left(x_{2}^{\prime}, \ldots, x_{l+1}^{\prime}, x, y_{1}, \ldots, y_{r}, z_{1}, \ldots, z_{t}\right)\right\} .
\end{aligned}
$$

Since there are $l$ alternatives from $X \backslash(Y(x) \cup Z(x) \cup\{x\})$ in the agendas $\left(x_{1}^{\prime}, x_{3}^{\prime}, \ldots, x_{l+1}^{\prime}\right.$, $\left.x, y_{1}, \ldots, y_{r}, z_{1}, \ldots, z_{t}\right)$ and $\left(x_{2}^{\prime}, \ldots, x_{l+1}^{\prime}, x, y_{1}, \ldots, y_{r}, z_{1}, \ldots, z_{t}\right)$, it follows that the outcome of both agendas is $x$. This proves (6).

Proof of Corollary 3.3. Let $P$ be asymmetric and let $x$ be an alternative such that there exists a maximal trajectory $\left(X^{\prime}, d\right)$ with $d(x)=t$, where $t=\# X^{\prime}$. Let $z_{l}=d^{-1}(l)$ for $l=1, \ldots, t-1$. Since $\left(X^{\prime}, d\right)$ is a trajectory, it follows that

$$
x P z_{l} \text { for all } l=1, \ldots, t-1
$$

and

$$
z_{l} P z_{k} \quad \text { for all } k=1, \ldots, t-2 \text { and for all } l \text { with } k<l \leq t-1 .
$$

Consider the set

$$
Y(x)=\{y \mid y P x\}^{24}
$$

If $Y(x)=\varnothing$, then by Theorem $3.1 x=o^{A}\left(x_{1}, \ldots, x_{m}\right)$ for some agenda. If $Y(x) \neq \varnothing$, then for all $y \in Y(x), \neg y P z_{l}$ for some $l \in\{1, \ldots, t-1\}$ since $\left(X^{\prime}, d\right)$ is a maximal trajectory. Since $x P z_{l}$ by (7), $z(y):=z_{l}$ fulfills condition (i) in Theorem 3.1. Moreover, if we let $Z(x)=\left\{z_{1}, \ldots, z_{t-1}\right\}$, then by (8) condition (ii) in Theorem 3.1 is satisfied for the ordering $\left(z_{t-1}, \ldots, z_{1}\right)$. Hence, $x=o^{A}\left(x_{1}, \ldots, x_{m}\right)$. This proves the first part of the claim.

\footnotetext{
${ }^{24}$ Observe that $y P x$ implies $\neg x P y$ since $P$ is asymmetric.
} 
Let $x=o^{A}\left(x_{1}, \ldots, x_{m}\right)$ for some agenda $\left(x_{1}, \ldots, x_{m}\right)$. If $Y(x)=\{y \mid y P x\}=\varnothing$, then $(\{x\}, d)$ with $d(x)=1$ is a maximal trajectory. If $Y(x) \neq \varnothing$, then Theorem 3.1 implies the existence of alternatives $z_{1}, \ldots, z_{t}$, such that the conditions (i) and (ii) in Theorem 3.1 are satisfied. Let $\left(z_{1}, \ldots, z_{t}\right)$ be the ordering of the alternatives such that (ii) holds. Then $\left(\left\{z_{1}, \ldots, z_{t}, x\right\}, d\right)$ with $d\left(z_{l}\right)=t-l+1$ for $l=1, \ldots, t$, and $d(x)=t+1$ is a trajectory. Let $y \notin\left\{z_{1}, \ldots, z_{t}, x\right\}$. Then either $\neg y P x$ or $y P x$. In the latter case, $y \in Y(x)$ and hence there exists an $l, 1 \leq l \leq t$, with $\neg y P z_{l}$. Hence, $\left(\left\{z_{1}, \ldots, z_{t}, x\right\}, d\right)$ is a maximal trajectory. This proves the claim.

Proof of Lemma 4.1. Let $\left(x_{1}, \ldots, x_{m}\right)$ be an agenda with $\bar{x}_{k} \neq x_{s}$ for some $s \geq k$. The proof is by backward induction over $l<k$. Let $l=k-1$. Then by definition $\bar{x}_{k-1} \in$ $\left\{x_{k-1}, \bar{x}_{k}\right\}$ and since $\bar{x}_{k} \neq x_{s}$ by assumption, it follows that $\bar{x}_{k-1} \neq x_{s}$. Suppose the claim has been proven for all $l$ with $t \leq l<k$, where $2 \leq t \leq k-1$. Since by definition $\bar{x}_{l-1} \in$ $\left\{x_{l-1}, \bar{x}_{l}\right\}$ and since $\bar{x}_{l} \neq x_{s}$ by assumption, it follows that $\bar{x}_{l-1} \neq x_{s}$.

Proof of Lemma 4.2. Let $x_{k}=o^{S}\left(x_{1}, \ldots, x_{m}\right)$ for some $1 \leq k \leq m$, and suppose by way of contradiction that $\bar{x}_{l} \neq x_{k}$ for some $l \leq k$. By Lemma 4.1 this implies that $\bar{x}_{s} \neq x_{k}$ for all $s<l$, contradicting the fact that $x_{k}=\bar{x}_{1}$.

Let $1 \leq k \leq m$ and let $\bar{x}_{l}=x_{k}$ for all $l \leq k$. In particular, we have $\bar{x}_{1}=o^{S}\left(x_{1}, \ldots, x_{m}\right)=$ $x_{k}$, which proves the claim.

Proof of Theorem 4.1. (i) Let $P$ be complete.

Necessity. Let $x_{k}=o^{S}\left(x_{1}, \ldots, x_{m}\right)$. Nothing has to be proved if $Y\left(x_{k}\right)=\varnothing$. Hence, let $Y\left(x_{k}\right) \neq \varnothing$ and let $Y\left(x_{k}\right)=\left\{x_{l(1)}, \ldots, x_{l(r)}\right\}$, where $l(1)<l(2)<\cdots<l(r)$. From Lemma 4.2 it follows that $k<l(1)$. We now construct a sequence $\left(z_{1}, \ldots, z_{t}\right)$ with the properties

- $z_{l+1} P z_{l}$ for all $l=1, \ldots, t-1$

- $x_{k} P z_{t}$

- $x \neq z_{s}$ for all $s=1, \ldots, t$

- for all $j=1, \ldots, r$, there exists an $s, 1 \leq s \leq t$, with $z_{s} P x_{l(j)}$.

Renumbering the alternatives such that $z_{s}^{\prime}=z_{t-s+1}$ for $s=1, \ldots, t$, and defining $Z\left(x_{k}\right)=$ $\left\{z_{1}^{\prime}, \ldots, z_{t}^{\prime}\right\}$, this will prove necessity.

Define $z_{1}=o^{S}\left(x_{l(r)}, x_{l(r)+1}, \ldots, x_{m}\right)$ and

$$
s(1)=\min \left\{h \mid o^{S}\left(x_{h}, \ldots, x_{m}\right)=z_{1}\right\} .
$$

Since $x_{k}=o^{S}\left(x_{1}, \ldots, x_{m}\right)$, it follows that $s(1)>k$.

Suppose $s(1) \leq l(1)$. Then $\neg x_{l(j)} P z_{1}$, which implies that $z_{1} P x_{l(j)}$ for all $j=1, \ldots, r-$ 1 , since $P$ is complete. If $x_{k} P z_{1}$ it follows that $z_{1} \neq x_{l(r)}$ and hence $\neg x_{l(r)} P z_{1}$, which implies that $z_{1} P x_{l(r)}$ since $P$ is complete. In this case we are done because the sequence $\left(z_{1}\right)$ has all the properties specified above. If $\neg x_{k} P z_{1}$, define $z_{2}=x_{s(1)-1}$. Then, by definition of $s(1), z_{2} P z_{1}$. Moreover, either $z_{1} \neq x_{l(r)}$ and hence $\neg x_{l(r)} P z_{1}$, which implies 
that $z_{1} P x_{l(r)}$ by completeness of $P$, or $z_{1}=x_{l(r)}$ and $z_{2} P x_{l(r)}$. If $x_{k} P z_{2}$ we are done because the sequence $\left(z_{1}, z_{2}\right)$ has all the properties specified above. If $\neg x_{k} P z_{2}$ there exists an $s(2)$ with $k<s(2)<s(1)$ such that $x_{s(2)} P z_{2}$. Let $z_{3}=x_{s(2)}$. If $x_{k} P z_{3}$ we are done because the sequence $\left(z_{1}, z_{2}, z_{3}\right)$ has all the properties specified above. Otherwise, we continue in the same manner. Since $x_{k}=o^{S}\left(x_{k}, \ldots, x_{m}\right)$, after finitely many steps we arrive at an alternative $z_{t}$ with $x_{k} P z_{t}$. The sequence $\left(z_{1}, \ldots, z_{t}\right)$ has all the properties specified above.

Suppose now that $l(j)<s(1) \leq l(j+1)$ for some $j$ with $1 \leq j \leq r-1$. Then $z_{1} P x_{l(i)}$ for all $i=j+1, \ldots, r-1$. Define $z_{2}=x_{s(1)-1}$. Then $z_{2} P z_{1}$ and either $z_{1}=x_{l(r)}$, which implies that $z_{2} P x_{l(r)}$, or $z_{1} \neq x_{l(r)}$, which implies that $\neg x_{l(r)} P z_{1}$ and hence $z_{1} P x_{l(r)}$ since $P$ is complete. Define

$$
s(2)=\min \left\{h \mid o^{S}\left(x_{h}, \ldots, x_{m}\right)=z_{2}\right\} .
$$

Observe that $s(2)<s(1)$. If $s(2) \leq l(1)$ we can use the same argument as in the case where $s(1) \leq l(1)$ to construct a sequence $\left(z_{1}, \ldots, z_{t}\right)$ with the desired properties. If $l(i)<$ $s(2) \leq l(i+1)$ for some $i$ with $1 \leq i \leq r-1$, define $z_{3}=x_{s(2)-1}$. Then $z_{3} P z_{2}$, and if $i<j$, then $\neg x_{l(h)} P z_{2}$, which implies that $z_{2} P x_{l(h)}$ for all $h=i+1, \ldots, j$, since $P$ is complete. Define

$$
s(3)=\min \left\{h \mid o^{S}\left(x_{h}, \ldots, x_{m}\right)=z_{3}\right\} .
$$

Again, either $s(3) \leq l(1)$ and we can follow the proof for the case where $s(1) \leq l(1)$ or $l(h)<s(3) \leq l(h+1)$ for some $h$ with $1 \leq h \leq r-1$. Continuing in this manner we see that after finitely many steps we arrive at an index $s(K)$ with $s(K) \leq l(1)$ and we can follow the argument in the proof for the case where $s(1) \leq l(1)$. This proves the existence of a sequence $\left(z_{1}, \ldots, z_{t}\right)$ with the desired properties.

Sufficiency. Let $x \in X$. If $Y(x)=\varnothing$, then by completeness of $P, x P y$ for all $y \neq x$. Hence, $x=o^{S}\left(x_{1}, \ldots, x_{m}\right)$ for any agenda $\left(x_{1}, \ldots, x_{m}\right)$ with $x_{1}=x$.

Let $Y(x) \neq \varnothing$. Then there exists a set of alternatives $Z(x)$ with $x \notin Z(x)$ and an ordering $\left(z_{1}, \ldots, z_{t}\right)$ of the alternatives in $Z(x)$ such that

- for all $y \in Y(x)$, there exists an $s, 1 \leq s \leq t$, with $z_{s} P y$

- $z_{l} P z_{l+1}$ for all $l=1, \ldots, t-1$

- $x P z_{1}$.

We now define an agenda $\left(w_{1}, \ldots, w_{r}\right)$ with $Y(x) \cup Z(x)=\left\{w_{1}, \ldots, w_{r}\right\}$ and $o^{S}\left(w_{1}\right.$, $\left.\ldots, w_{r}\right)=z_{1}$. Let $Y^{\prime}=Y(x) \backslash Z(x)$. If $Y^{\prime}=\varnothing$, then let $r=t$ and $w_{s}=z_{s}$ for all $s=1, \ldots, t$. In this case it immediately follows that $o^{S}\left(w_{1}, \ldots, w_{r}\right)=z_{1}$. If $Y^{\prime} \neq \varnothing$, let $\left(w_{1}, \ldots, w_{r}\right)$ be the agenda that is obtained if all $y \in Y^{\prime}$ with $z_{t} P y$ (if any) are placed after $z_{t}$, all $y \in Y^{\prime}$ with $\neg z_{t} P y$ and $z_{t-1} P y$ (if any) are placed between $z_{t-1}$ and $z_{t}$, and so on, and finally all $y \in Y^{\prime}$ with $\neg z_{s} P y$ for all $s=2, \ldots, t$, and $z_{1} P y$ (if any) are placed between $z_{1}$ and $z_{2}$. Then, by definition $Y(x) \cup Z(x)=\left\{w_{1}, \ldots, w_{r}\right\}$ and $o^{S}\left(w_{1}, \ldots, w_{r}\right)=z_{1}$.

If $Y(x) \cup Z(x) \cup\{x\}=X$, then

$$
x=o^{S}\left(x, w_{1}, \ldots, w_{r}\right)
$$


since $x P z_{1}$. If $X \backslash(Y(x) \cup Z(x) \cup\{x\})=\left\{x_{1}, \ldots, x_{m-r-1}\right\}$, where $r \leq m-2$, then $x P x_{s}$ for all $s=1, \ldots, m-r-1$, since $P$ is complete. Since

$$
o^{S}\left(x_{1}, \ldots, x_{m-r-1}, w_{1}, \ldots, w_{r}\right) \in\left\{x_{1}, \ldots, x_{m-r-1}, z_{1}\right\},
$$

it follows that $x P o^{S}\left(x_{1}, \ldots, x_{m-r-1}, w_{1}, \ldots, w_{r}\right)$, which implies that

$$
x=o^{S}\left(x, x_{1}, \ldots, x_{m-r-1}, w_{1}, \ldots, w_{r}\right) .
$$

This proves sufficiency.

(ii) Let $P$ be asymmetric. The proof is very similar to the proof for a complete relation $P$.

Necessity. Let $x_{k}=o^{S}\left(x_{1}, \ldots, x_{m}\right)$. Nothing has to be proved if $Y\left(x_{k}\right)=\varnothing$. Hence, let $Y\left(x_{k}\right) \neq \varnothing$ and let $Y\left(x_{k}\right)=\left\{x_{l(1)}, \ldots, x_{l(r)}\right\}$, where $l(1)<l(2)<\cdots<l(r)$. From Lemma 4.2 it follows that $k<l(1)$. We now construct a sequence $\left(z_{1}, \ldots, z_{t}\right)$ with the properties

- $z_{l+1} P z_{l}$ for all $l=1, \ldots, t-1$

- $x_{k} P z_{t}$

- $x \neq z_{s}$ for all $s=1, \ldots, t$

- for all $j=1, \ldots, r$, there exists an $s, 1 \leq s \leq t$, with $\neg x_{l(j)} P z_{s}$.

Again, renumbering the alternatives such that $z_{s}^{\prime}=z_{t-s+1}$ for $s=1, \ldots, t$ and defining $Z\left(x_{k}\right)=\left\{z_{1}^{\prime}, \ldots, z_{t}^{\prime}\right\}$, this will prove necessity.

Define $z_{1}=o^{S}\left(x_{l(r)}, x_{l(r)+1}, \ldots, x_{m}\right)$ and

$$
s(1)=\min \left\{h \mid o^{S}\left(x_{h}, \ldots, x_{m}\right)=z_{1}\right\} .
$$

Since $x_{k}=o^{S}\left(x_{1}, \ldots, x_{m}\right)$ it follows that $s(1)>k$.

Suppose $s(1) \leq l(1)$. Then $\neg x_{l(j)} P z_{1}$ for all $j=1, \ldots, r-1$. If $x_{k} P z_{1}$, it follows that $z_{1} \neq x_{l(r)}$ and hence $\neg x_{l(r)} P z_{1}$. In this case we are done because the sequence $\left(z_{1}\right)$ has all the properties specified above. If $\neg x_{k} P z_{1}$, define $z_{2}=x_{s(1)-1}$. Then $z_{2} P z_{1}$ and either $z_{1} \neq x_{l(r)}$, which implies that $\neg x_{l(r)} P z_{1}$, or $z_{1}=x_{l(r)}$ and hence $z_{2} P x_{l(r)}$, which implies that $\neg x_{l(r)} P z_{2}$ since $P$ is asymmetric. If $x_{k} P z_{2}$, we are done because the sequence $\left(z_{1}, z_{2}\right)$ has all the properties specified above. If $\neg x_{k} P z_{2}$, there exists an $s(2)$ with $k<s(2)<s(1)$ such that $x_{s(2)} P z_{2}$. Let $z_{3}=x_{s(2)}$. If $x_{k} P z_{3}$, we are done because the sequence $\left(z_{1}, z_{2}, z_{3}\right)$ has all the properties specified above; otherwise, we continue in the same manner. Since $x_{k}=o^{S}\left(x_{k}, \ldots, x_{m}\right)$, after finitely many steps we arrive at an alternative $z_{t}$ with $x_{k} P z_{t}$. The sequence $\left(z_{1}, \ldots, z_{t}\right)$ has all the properties specified above.

Suppose now that $l(j)<s(1) \leq l(j+1)$ for some $j$ with $1 \leq j \leq r-1$. Then $\neg x_{l(i)} P z_{1}$ for all $i=j+1, \ldots, r-1$. Define $z_{2}=x_{s(1)-1}$. Then $z_{2} P z_{1}$ and either $z_{1}=x_{l(r)}$, which implies that $z_{2} P x_{l(r)}$ and hence $\neg x_{l(r)} P z_{2}$ since $P$ is asymmetric, or $z_{1} \neq x_{l(r)}$, which implies that $\neg x_{l(r)} P z_{1}$. Define

$$
s(2)=\min \left\{h \mid o^{S}\left(x_{h}, \ldots, x_{m}\right)=z_{2}\right\} .
$$


Observe that $s(2)<s(1)$. If $s(2) \leq l(1)$, we can use the same argument as in the case where $s(1) \leq l(1)$ to construct a sequence $\left(z_{1}, \ldots, z_{t}\right)$ with the desired properties. If $l(i)<$ $s(2) \leq l(i+1)$ for some $i$ with $1 \leq i \leq r-1$, define $z_{3}=x_{s(2)-1}$. Then $z_{3} P z_{2}$ and if $i<j$, then $\neg x_{l(h)} P z_{2}$ for all $h=i+1, \ldots, j$. Define

$$
s(3)=\min \left\{h \mid o^{S}\left(x_{h}, \ldots, x_{m}\right)=z_{3}\right\} .
$$

Again, either $s(3) \leq l(1)$ and we can follow the proof for the case where $s(1) \leq l(1)$, or $l(h)<s(3) \leq l(h+1)$ for some $h$ with $1 \leq h \leq r-1$. Continuing in this manner we see that after finitely many steps we arrive at an index $s(K)$ with $s(K) \leq l(1)$ and we can follow the argument in the proof for the case where $s(1) \leq l(1)$. This proves the existence of a sequence $\left(z_{1}, \ldots, z_{t}\right)$ with the desired properties.

Sufficiency. Let $x \in X$. If $Y(x)=\varnothing$, then $\neg y P x$ for all $y \neq x$. Hence, $x=$ $o^{S}\left(x_{1}, \ldots, x_{m}\right)$ for any agenda $\left(x_{1}, \ldots, x_{m}\right)$ with $x_{m}=x$.

Let $Y(x) \neq \varnothing$. Then there exists a set of alternatives $Z(x)$ with $x \notin Z(x)$ and an ordering $\left(z_{1}, \ldots, z_{t}\right)$ of the alternatives in $Z(x)$ such that

- for all $y \in Y(x)$, there exists an $s, 1 \leq s \leq t$, with $\neg y P z_{s}$

- $z_{l} P z_{l+1}$ for all $l=1, \ldots, t-1$

- $x P z_{1}$.

We now define an agenda $\left(w_{1}, \ldots, w_{r}\right)$ with $Y(x) \cup Z(x)=\left\{w_{1}, \ldots, w_{r}\right\}$ and $o^{S}\left(w_{1}\right.$, $\left.\ldots, w_{r}\right)=z_{1}$. Let $Y^{\prime}=Y(x) \backslash Z(x)$. If $Y^{\prime}=\varnothing$, then let $r=t$ and $w_{s}=z_{s}$ for all $s=1, \ldots, t$. In this case it immediately follows that $o^{S}\left(w_{1}, \ldots, w_{r}\right)=z_{1}$. If $Y^{\prime} \neq \varnothing$, let $\left(w_{1}, \ldots, w_{r}\right)$ be the agenda that is obtained if all $y \in Y^{\prime}$ with $\neg y P z_{t}$ (if any) are placed between $z_{t-1}$ and $z_{t}$, all $y \in Y^{\prime}$ with $y P z_{t}$ and $\neg y P z_{t-1}$ (if any) are placed between $z_{t-2}$ and $z_{t-1}$, and so on, and finally all $y \in Y^{\prime}$ with $y P z_{s}$ for all $s=2, \ldots, t$ and $\neg y P z_{1}$ (if any) are placed before $z_{1}$. Then, by definition $Y(x) \cup Z(x)=\left\{w_{1}, \ldots, w_{r}\right\}$ and $o^{S}\left(w_{1}, \ldots, w_{r}\right)=z_{1}$.

If $Y(x) \cup Z(x) \cup\{x\}=X$, then

$$
x=o^{S}\left(x, w_{1}, \ldots, w_{r}\right)
$$

since $x P z_{1}$. If $X \backslash(Y(x) \cup Z(x) \cup\{x\})=\left\{x_{1}, \ldots, x_{m-r-1}\right\}$, where $r \leq m-2$, then $\neg x_{s} P x$ for all $s=1, \ldots, m-r-1$, which implies that

$$
x=o^{S}\left(x_{1}, \ldots, x_{m-r-1}, x, w_{1}, \ldots, w_{r}\right) .
$$

This proves sufficiency.

Proof of Corollary 4.2. Let $P$ be complete. Assume first that $x=o^{S}\left(x_{1}, \ldots, x_{m}\right)$ for some agenda $\left(x_{1}, \ldots, x_{m}\right)$ and let $y \in X, y \neq x$. If $x P y$, then define $z_{0}=x$ and $z_{1}=y$. If $\neg x P y$, then $y P x$ by completeness of $P$. In this case, by Theorem 4.1 there exists a sequence of alternatives $\left(z_{1}, \ldots, z_{t}\right)$ with the properties

- there exists an $s, 1 \leq s \leq t$, such that $z_{s} P y$ 
- $z_{l} P z_{l+1}$ for all $l=1, \ldots, t-1$

- $x P z_{1}$.

This proves that $x$ is in the top cycle of $P$.

For the reverse, let $x$ be in the top cycle of $P$ and let $Y(x)=\{y \mid y P x$ and $\neg x P y\}$. If $Y(x)=\varnothing$, then $x=o^{S}\left(x_{1}, \ldots, x_{m}\right)$ for some agenda $\left(x_{1}, \ldots, x_{m}\right)$ by Theorem 4.1 and we are done. Suppose $Y(x)=\left\{y_{1}, \ldots, y_{r}\right\}$ for some $r \geq 1$. Since $x$ is in the top cycle of $P$, for all $l=1, \ldots, r$, there exists a sequence of distinct alternatives $\left(w_{1}^{l}, \ldots, w_{s(l)}^{l}\right)$ with $w_{1}^{l}=y_{l}, w_{k+1}^{l} P w_{k}^{l}$ for all $k=1, \ldots, s(l)$, and $x P w_{s(l)}^{l}$.

For $l=1, \ldots, r$, we now inductively define sequences of distinct alternatives $z^{l}$ as follows: For $l=1$, define $\bar{s}(1)=s(1)$ and

$$
z^{1}=\left(w_{\bar{s}(1)}^{1}, \ldots, w_{1}^{1}\right) .
$$

For $l=2$, let $z^{2}=z^{1}$, if $y_{2} \in\left\{w_{1}^{1}, \ldots, w_{\bar{s}(1)}^{1}\right\}$. Otherwise, if $y_{2} \notin\left\{w_{1}^{1}, \ldots, w_{\bar{s}(1)}^{1}\right\}$ let $\bar{s}(2) \in$ $\{1, \ldots, s(2)-1\}$ be the minimal $s$ with the property that

$$
w_{s+1}^{2} \in\left\{w_{1}^{1}, \ldots, w_{\bar{s}(1)}^{1}\right\} .
$$

If there is no such $s$ define $\bar{s}(2)=s(2)$. Then define

$$
z^{2}=\left(w_{\bar{s}(1)}^{1}, \ldots, w_{1}^{1}, w_{\bar{s}(2)}^{2}, \ldots, w_{1}^{2}\right) .
$$

For ease of presentation we assume that $y_{2} \notin\left\{w_{1}^{1}, \ldots, w_{\bar{s}(1)}^{1}\right\}$ and then continue to define $z^{3}$. If $y_{3} \in\left\{w_{1}^{1}, \ldots, w_{\bar{s}(1)}^{1}, w_{1}^{2}, \ldots, w_{\bar{s}(2)}^{2}\right\}$, define $z^{3}=z^{2}$. Otherwise, if $y_{2} \notin$ $\left\{w_{1}^{1}, \ldots, w_{\bar{s}(1)}^{1}, w_{1}^{2}, \ldots, w_{\bar{s}(2)}^{2}\right\}$, let $\bar{s}(3) \in\{1, \ldots, s(3)-1\}$ be the minimal $s$ with the property that

$$
w_{s+1}^{3} \in\left\{w_{1}^{1}, \ldots, w_{\bar{s}(1)}^{1}, w_{1}^{2}, \ldots, w_{\bar{s}(2)}^{2}\right\} .
$$

If there is no such $s$, define $\bar{s}(3)=s(3)$. Then define

$$
z^{3}=\left(w_{\bar{s}(1)}^{1}, \ldots, w_{1}^{1}, w_{\bar{s}(2)}^{2}, \ldots, w_{1}^{2}, w_{\bar{s}(3)}^{3}, \ldots, w_{1}^{3}\right) .
$$

Continuing in this manner and assuming that $y_{k} \notin \bigcup_{l=1}^{k-1}\left\{w_{1}^{l}, \ldots, w_{\bar{s}(l)}^{l}\right\}$ for all $k=$ $2, \ldots, r,{ }^{25}$ we arrive at the sequence

$$
z^{r}=\left(w_{\bar{s}(1)}^{1}, \ldots, w_{1}^{1}, w_{\bar{S}(2)}^{2}, \ldots, w_{1}^{2}, \ldots, w_{\bar{s}(r)}^{r}, \ldots, w_{1}^{r}\right) .
$$

Observe that by construction $z^{r}$ has the property that $y_{k} \in \bigcup_{l=1}^{r}\left\{w_{1}^{l}, \ldots, w_{\bar{s}(l)}^{l}\right\}$ for all $k=$ $1, \ldots, r$ and that

$$
o^{S}\left(w_{\bar{s}(1)}^{1}, \ldots, w_{1}^{1}, w_{\bar{s}(2)}^{2}, \ldots, w_{1}^{2}, \ldots, w_{\bar{s}(r)}^{r}, \ldots, w_{1}^{r}\right) \in\left\{w_{s(1)}^{1}, w_{s(2)}^{2}, \ldots, w_{s(r)}^{r}\right\} .
$$

\footnotetext{
${ }^{25}$ The proof for the case where $y_{k} \in \bigcup_{l=1}^{k-1}\left\{w_{1}^{l}, \ldots, w_{\bar{s}(l)}^{l}\right\}$ for some $k$ and hence, $z^{k}=z^{k-1}$ is similar and hence is omitted.
} 
Since $x P w_{s(l)}^{l}$ for all $l=1, \ldots, r$, it follows that

$$
x=o^{S}\left(x, w_{\bar{s}(1)}^{1}, \ldots, w_{1}^{1}, w_{\bar{s}(2)}^{2}, \ldots, w_{1}^{2}, \ldots, w_{\bar{s}(r)}^{r}, \ldots, w_{1}^{r}\right) .
$$

If $X \backslash\{x\}=\bigcup_{l=1}^{r}\left\{w_{1}^{l}, \ldots, w_{\bar{s}(l)}^{l}\right\}$, we are done. Otherwise, let

$$
X \backslash\left(\{x\} \cup \bigcup_{l=1}^{r}\left\{w_{1}^{l}, \ldots, w_{\bar{s}(l)}^{l}\right\}\right)=\left\{x_{1}, \ldots, x_{t}\right\} .
$$

Since $P$ is complete and $Y(x) \subset \bigcup_{l=1}^{r}\left\{w_{1}^{l}, \ldots, w_{\bar{s}(l)}^{l}\right\}$, it follows that $x P x_{s}$ for all $s=$ $1, \ldots, t$. Hence,

$$
x=o^{S}\left(x, x_{1}, \ldots, x_{t}, w_{\bar{s}(1)}^{1}, \ldots, w_{1}^{1}, w_{\bar{s}(2)}^{2}, \ldots, w_{1}^{2}, \ldots, w_{\bar{s}(r)}^{r}, \ldots, w_{1}^{r}\right) .
$$

This proves the claim that any alternative $x$ in the top cycle is an outcome for some agenda under the successive procedure.

Proof of Theorem 5.1. The theorem is proved by showing that (i) and (ii) are equivalent to (iii).

(i) $\Longleftrightarrow$ (iii). Assume (iii), i.e., $x P y$ and $\neg y P x$ for all $y \neq x$. Then, by Theorem 4.1, $O^{S}(P)=\{x\}$, i.e., (i) holds. Assume (i), i.e., $O^{S}(P)=\{x\}$. Then, $x=o^{S}\left(x_{1}, \ldots, x_{m}\right)$ for any agenda with $x_{m}=x$. By definition of the successive procedure, this implies that $x=o^{S}\left(x_{k}, \ldots, x_{m}\right)$ for all $k=1, \ldots, m-1$ and hence $\neg x_{k} P x$ for all $k=1, \ldots, m-1$. This already establishes the proof when $P$ is complete. As for the asymmetric case, suppose by way of contradiction that $\neg x P y$ for some $y \neq x$. Then $o^{S}(x, y)=y$, which implies that $x \neq o^{S}\left(x_{1}, \ldots, x_{m}\right)$ for any agenda with $x_{m-1}=x$ and $x_{m}=y$ by Lemma 4.1. This contradicts our assumption that $O^{S}(P)=\{x\}$. Hence, also for $P$ asymmetric, $O^{S}(P)=\{x\}$ implies that (iii) holds.

(ii) $\Longleftrightarrow$ (iii). Assume (iii), i.e., $x P y$ and $\neg y P x$ for all $y \neq x$. Then, by Theorem 3.1, $O^{A}(P)=\{x\}$, i.e., (ii) holds. Assume (ii), i.e., $O^{A}(P)=\{x\}$. First consider the case where $P$ is asymmetric. Then $x=o^{A}\left(x_{1}, \ldots, x_{m}\right)$ for all agendas $\left(x_{1}, \ldots, x_{m}\right)$. Let $y \in X, y \neq$ $x$. Then $x=o^{A}\left(x, x_{1}, \ldots, x_{m-2}, y\right)$, where $\left(x_{1}, \ldots, x_{m-2}\right)$ is an arbitrary ordering of the alternatives different from $x$ and $y$. Lemma 3.1 then implies that $x P y$. Hence, $O^{A}(P)=$ $\{x\}$ implies that $x P y$ for all $y \neq x$. Since $P$ is asymmetric, this implies (iii).

Next consider the case where $P$ is complete. Suppose by way of contradiction that there exists $y \in X, y \neq x$, with $y P x$. We then claim that $x \neq o^{A}\left(x_{1}, \ldots, x_{m}\right)$ for any agenda with $x_{1}=y$ and $x_{m}=x$. The claim is proved by induction over $m$. If $m=2$, the claim is immediate. Suppose now that the claim is true for $m \geq 2$ and consider the agenda $\left(x_{1}, \ldots, x_{m+1}\right)$ with $x_{1}=y$ and $x_{m+1}=x$. Suppose by way of contradiction that $x=o^{A}\left(x_{1}, \ldots, x_{m+1}\right)$. By definition of the amendment procedure,

$$
o^{A}\left(x_{1}, \ldots, x_{m+1}\right) \in\left\{o^{A}\left(x_{1}, x_{3}, \ldots, x_{m+1}\right), o^{A}\left(x_{2}, \ldots, x_{m+1}\right)\right\} .
$$

Since the agenda $\left(x_{1}, x_{3}, \ldots, x_{m+1}\right)$ has $m$ alternatives, it follows that $x \neq o^{A}\left(x_{1}, x_{3}\right.$, $\left.\ldots, x_{m+1}\right)$. Then $x=o^{A}\left(x_{1}, \ldots, x_{m+1}\right)$ implies that $x=o^{A}\left(x_{2}, \ldots, x_{m+1}\right)$ and

$$
\neg O^{A}\left(x_{1}, x_{3}, \ldots, x_{m+1}\right) P x .
$$


Since $x_{1}=y$ and $y P x$ it follows that $o^{A}\left(x_{1}, x_{3}, \ldots, x_{m+1}\right)=x_{k}$ for some $k$ with $3 \leq k \leq m$. Lemma 3.2 then implies that $x_{k}=o^{A}\left(x_{k}, \ldots, x_{m+1}\right)=\bar{x}_{k}$. But then $x_{k} P \bar{x}_{m+1}$ by Lemma 3.1. Since $\bar{x}_{m+1}=x_{m+1}=x$, this is a contradiction to (9). This proves our claim.

Hence, $O^{A}(P)=\{x\}$ implies that $\neg y P x$ for all $y \neq x$. Finally, by completeness of $P$, we conclude that $x P y$ for all $y \neq x$, i.e., (iii) holds.

Proof of Proposition 5.2. Consider first the case where $1 \leq q<q^{\prime} \leq\left\lfloor\frac{n}{2}\right\rfloor+1$ and let $\mathcal{P} \in \Phi(q)$. Then, by definition of $\Phi(q)$, there exists an alternative $x$ such that for all $y \neq x$,

$$
\#\left\{i \mid x \mathcal{P}_{i} y\right\} \geq q \quad \text { and } \quad \#\left\{i \mid y \mathcal{P}_{i} x\right\}<q .
$$

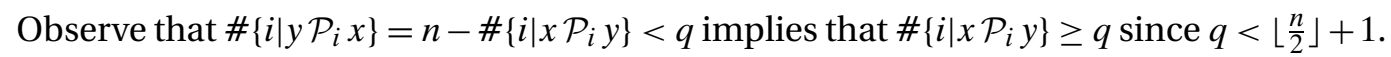
Hence, (10) is satisfied if and only if

$$
\#\left\{i \mid x \mathcal{P}_{i} y\right\}>n-q
$$

This immediately implies that $\Phi(q) \subseteq \Phi\left(q^{\prime}\right)$ if $q<q^{\prime}<\left\lfloor\frac{n}{2}\right\rfloor+1$. It remains to consider the case where $q=\left\lfloor\frac{n}{2}\right\rfloor$ and $q^{\prime}=\left\lfloor\frac{n}{2}\right\rfloor+1$. If $n$ is odd, then by what we have shown above, $\mathcal{P} \in \Phi\left(\left\lfloor\frac{n}{2}\right\rfloor\right)$ if and only if there exists an alternative $x$ such that for all $y \neq x$,

$$
\#\left\{i \mid x \mathcal{P}_{i} y\right\}>n-\left\lfloor\frac{n}{2}\right\rfloor=\frac{n+1}{2}=\left\lfloor\frac{n}{2}\right\rfloor+1 .
$$

This implies

$$
\#\left\{i \mid y \mathcal{P}_{i} x\right\}<\frac{n-1}{2}=\left\lfloor\frac{n}{2}\right\rfloor<\left\lfloor\frac{n}{2}\right\rfloor+1 .
$$

Hence, $\mathcal{P} \in \Phi\left(\left\lfloor\frac{n}{2}\right\rfloor+1\right)$, i.e., $\Phi\left(\left\lfloor\frac{n}{2}\right\rfloor\right) \subseteq \Phi\left(\left\lfloor\frac{n}{2}\right\rfloor+1\right)$. If $n$ is even, then by the above, $\mathcal{P} \in$ $\Phi\left(\left\lfloor\frac{n}{2}\right\rfloor\right)$ if and only if there exists an alternative $x$ such that for all $y \neq x$,

$$
\#\left\{i \mid x \mathcal{P}_{i} y\right\}>n-\left\lfloor\frac{n}{2}\right\rfloor=\frac{n}{2} .
$$

This implies

$$
\#\left\{i \mid x \mathcal{P}_{i} y\right\} \geq \frac{n}{2}+1
$$

and

$$
\#\left\{i \mid y \mathcal{P}_{i} x\right\}<\frac{n}{2}<\frac{n}{2}+1 .
$$

Hence, also for $n$ even we conclude that $\mathcal{P} \in \Phi\left(\left\lfloor\frac{n}{2}\right\rfloor+1\right)$, which implies that $\Phi\left(\left\lfloor\frac{n}{2}\right\rfloor\right) \subseteq$ $\Phi\left(\left\lfloor\frac{n}{2}\right\rfloor+1\right)$.

Next consider the case where $\left\lfloor\frac{n}{2}\right\rfloor+1 \leq q^{\prime}<q \leq n$ and let $\mathcal{P} \in \Phi(q)$. Since the majority relation is asymmetric for quotas greater than or equal to $\left\lfloor\frac{n}{2}\right\rfloor+1$, by definition of $\Phi(q)$ there exists a unique alternative $x$ such that for all $y \neq x$,

$$
\#\left\{i \mid x \mathcal{P}_{i} y\right\} \geq q
$$

Since $q>q^{\prime}$ this immediately implies that $\Phi(q) \subseteq \Phi\left(q^{\prime}\right)$. 
Proof of Proposition 5.3. Suppose by way of contradiction that there exists a $q \in$ $\{1, \ldots, n-1\}$ and an alternative $x \in X$ such that $x \in O^{A}(\mathcal{P}, q)$ and $x \notin O^{A}(\mathcal{P}, n)$. By Theorem 3.1 the latter implies that there exists an alternative $y$ with $y \mathcal{P}_{i} x$ for all $i=1, \ldots, n$. Since $x \in O^{A}(\mathcal{P}, q)$, by Theorem 3.1 there exists $z(y)$ with

$$
\begin{aligned}
& \#\left\{i \mid y \mathcal{P}_{i} z(y)\right\}<q \text { and } \\
& \#\left\{i \mid x \mathcal{P}_{i} z(y)\right\} \geq q .
\end{aligned}
$$

However, $y \mathcal{P}_{i} x$ for all $i=1, \ldots, n$ and (12) imply that $\#\left\{i \mid y \mathcal{P}_{i} z(y)\right\} \geq q$, contradicting (11). This proves the claim that $O^{A}(\mathcal{P}, q) \subseteq O^{A}(\mathcal{P}, n)$.

Proof of Proposition 5.4. (i) For $x \in X$ and $q \in\{1, \ldots, n\}$, define

$$
Y_{q}(x):=\left\{y \mid \#\left\{i \mid y \mathcal{P}_{i} x\right\} \geq q \text { and } \#\left\{i \mid x \mathcal{P}_{i} y\right\}<q\right\}
$$

Let $q, q^{\prime}$ be given with $1 \leq q<q^{\prime} \leq\left\lfloor\frac{n}{2}\right\rfloor+1$. Then the dominance relation $P$ derived from majority voting with quota $q$ is complete. Let $x \in O^{S}\left(\mathcal{P}, q^{\prime}\right)$. If $Y_{q}(x)=\varnothing$, then by Theorem 4.1, $x \in O^{S}(\mathcal{P}, q)$ and we are done. It remains to consider the case where $Y_{q}(x) \neq \varnothing$. We will first prove that

$$
Y_{q}(x) \subseteq Y_{q^{\prime}}(x)
$$

Let $y \in Y_{q}(x)$. Then $\#\left\{i \mid x \mathcal{P}_{i} y\right\}<q<q^{\prime}$, which implies that

$$
\#\left\{i \mid y \mathcal{P}_{i} x\right\}>n-q
$$

Since $q<\left\lfloor\frac{n}{2}\right\rfloor+1$, (14) implies that

$$
\#\left\{i \mid y \mathcal{P}_{i} x\right\} \geq\left\lfloor\frac{n}{2}\right\rfloor+1 \geq q^{\prime} .
$$

Hence, from (14) it follows that $\#\left\{i \mid y \mathcal{P}_{i} x\right\} \geq q^{\prime}$, which implies that $y \in Y_{q^{\prime}}(x)$. This proves (13).

Consider first the case where either $n$ is even and $q^{\prime} \leq \frac{n}{2}$ or $n$ is odd and $q^{\prime} \leq$ $\left\lfloor\frac{n}{2}\right\rfloor+1$. In both cases the dominance relation $P$ derived from majority voting with quota $q^{\prime}$ is complete. Since $x \in O^{S}\left(\mathcal{P}, q^{\prime}\right)$, Theorem 4.1 implies that there exists a sequence of distinct alternatives $\left(z_{1}, \ldots, z_{t}\right)$ with the properties

- for all $y \in Y_{q^{\prime}}(x)$, there exists an $s, 1 \leq s \leq t$, with $\#\left\{i \mid z_{s} \mathcal{P}_{i} y\right\} \geq q^{\prime}$

- \#\{i|zl $\left.\mathcal{P}_{i} z_{l+1}\right\} \geq q^{\prime}$ for all $l=1, \ldots, t-1$

- $\#\left\{i \mid x \mathcal{P}_{i} z_{1}\right\} \geq q^{\prime}$.

Since $q^{\prime}>q$, (13) and Theorem 4.1 imply that $x \in O^{S}(\mathcal{P}, q)$. It remains to consider the case where $n$ is even and $q^{\prime}=\frac{n}{2}+1$. In this case, the dominance relation derived from majority voting with quota $q^{\prime}$ is asymmetric (but not complete) and Theorem 4.1 implies that there exists a sequence of distinct alternatives $\left(z_{1}, \ldots, z_{t}\right)$ with the properties 
- for all $y \in Y_{q^{\prime}}(x)$, there exists an $s, 1 \leq s \leq t$, with \#\{i|y $\left.\mathcal{P}_{i} z_{s}\right\}<q^{\prime}$

- \#\{i|zl $\left.\mathcal{P}_{i} z_{l+1}\right\} \geq q^{\prime}$ for all $l=1, \ldots, t-1$

- \#\{i|x $\left.\mathcal{P}_{i} z_{1}\right\} \geq q^{\prime}$.

Since $q^{\prime}=\frac{n}{2}+1$, the first property implies that for all $y \in Y_{q^{\prime}}(x)$ there exists an $s, 1 \leq s \leq t$, with \#\{i|zs $\left.\mathcal{P}_{i} y\right\} \geq \frac{n}{2} \geq q$. But then as above we can use (13) and Theorem 4.1 to conclude that $x \in O^{S}(\mathcal{P}, q)$ for $q<q^{\prime}$ since the dominance relation $P$ derived from majority voting with quota $q$ is complete. This proves the claim.

Proof of Proposition 5.5.

(i) Let $q \in\{1, \ldots, n\}$ and let $x \in O^{A}(\mathcal{P}, q)$. Suppose by way of contradiction that $x$ is Pareto dominated by some alternative $y$, i.e., $y \mathcal{P}_{i} x$ for all voters $i$. Then $y \in Y(x)$ and by Theorem 3.1 there exists an alternative $z(y)$ such that

$$
\begin{aligned}
& \#\left\{i \mid y \mathcal{P}_{i} z(y)\right\}<q \text { and } \\
& \#\left\{i \mid x \mathcal{P}_{i} z(y)\right\} \geq q .
\end{aligned}
$$

However, since $y \mathcal{P}_{i} x$ for all voters $i$, (16) implies that \#\{i|y $\left.\mathcal{P}_{i} z(y)\right\} \geq q$, contradicting (15). Hence, $x$ is not Pareto dominated by any alternative $y$.

(ii) Let $x \in X$ and let $q \in\{1, n\}$. Then $Y(x)=\left\{y \mid y \mathcal{P}_{i} x\right.$ for all $\left.i\right\}$, i.e., $Y(x)$ is the set of all alternatives that Pareto dominate $x$. From (i) we know that $O^{A}(\mathcal{P}, q) \subseteq$ $\left\{x \mid\right.$ there exists no $y$ with $y \mathcal{P}_{i} x$ for all $\left.i\right\}$. Hence, it remains to show that any alternative $x$, which is not Pareto dominated, is an element of $O^{A}(\mathcal{P}, q)$. If $x$ is not Pareto dominated by any other alternative, then $Y(x)=\varnothing$ and Theorem $3.1 \mathrm{im}$ plies that $x \in O^{A}(\mathcal{P}, q)$.

Proof of Proposition 5.6. (i) If $q=n$, then $Y(x)=\left\{y \mid y \mathcal{P}_{i} x\right.$ for all $\left.i\right\}$. Hence, if $x$ is an alternative that is not Pareto dominated by any other alternative, then $Y(x)=\varnothing$, and Theorem 4.1 implies that $x \in O^{S}(\mathcal{P}, n)$. Now let $x \in O^{S}(\mathcal{P}, n)$ and suppose by way of contradiction that $x$ is Pareto dominated by $y$. Then $y \in Y(x)$ and by Theorem 4.1 there exists a set of alternatives $Z(x)$ and an ordering $\left(z_{1}, \ldots, z_{t}\right)$ of the alternatives in $Z(x)$ such that the following conditions are satisfied:

- There exists an $s \in\{1, \ldots, t\}$ and a voter $j$ with $z_{s} \mathcal{P}_{j} y$.

- We have $z_{l} \mathcal{P}_{i} z_{l+1}$ for all voters $i$ and for all $l=1, \ldots, t-1$.

- We have $x \mathcal{P}_{i} z_{1}$ for all voters $i$.

However, this implies that

$$
x \mathcal{P}_{j} z_{1} \mathcal{P}_{j} z_{2} \cdots \mathcal{P}_{j} z_{s} \mathcal{P}_{j} y,
$$

contradicting the assumption that $y$ Pareto dominates $x$. Hence, no alternative in $O^{S}(\mathcal{P}, n)$ is Pareto dominated, which proves the claim. 


\section{REFERENCES}

Apesteguia, Jose, Miguel A. Ballester, and Yusufcan Masatlioglu (2014), "A foundation for strategic agenda voting." Games and Economic Behavior, 87, 91-99. [212]

Austen-Smith, David and Jeffrey S. Banks (2005), Positive Political Theory II: Strategy and Structure. University of Michigan Press, Ann Arbor. [215, 217]

Banks, Jeffrey S. (1985), "Sophisticated voting outcomes and agenda control." Social Choice and Welfare, 1, 295-306. [212, 214, 217]

Banks, Jeffrey S. and Georges A. Bordes (1988), "Voting games, indifference, and consistent sequential choice rules." Social Choice and Welfare, 5, 31-44. [214, 223, 230]

Barberà, Salvador and Anke Gerber (2015), Sequential voting and agenda manipulation: The case of forward looking tie-breaking. Barcelona GSE Working Paper Series No. 782, Universitat Autònoma de Barcelona. [230]

Duggan, John (2006), “Endogenous voting agendas.” Social Choice and Welfare, 27, 495530. [212]

Dutta, Bhaskar, Matthew O. Jackson, and Michel Le Breton (2004), "Equilibrium agenda formation." Social Choice and Welfare, 23, 21-37. [212]

Farquharson, Robin (1969), Theory of Voting. Yale University Press, New Haven. [212, 216]

Gretlein, Rodney J. (1982), “Dominance solvable voting schemes: A comment.” Econometrica, 50, 527-528. [217]

Laslier, Jean-François (1997), Tournament Solutions and Majority Voting. Springer, Berlin. [214, 217, 228]

McKelvey, Richard D. and Richard G. Niemi (1978), "A multistage game represenation of sophisticated voting for binary procedures.” Journal of Economic Theory, 18, 1-22. [217]

McLean, Iain and Arnold B. Urken (1995), Classics of Social Choice. University of Michigan Press. [212]

Miller, Nicholas R. (1977), "Graph-theoretical approaches to the theory of voting." American Journal of Political Science, 21, 769-803. [212, 214, 224, 228]

Miller, Nicholas R. (1980), "A new solution set for tournaments and majority voting: Further graph-theoretical approaches to the theory of voting." American Journal of Political Science, 24, 68-96. [212, 214, 222, 223, 228]

Miller, Nicholas R. (1995), “Committees, agendas, and voting.” In Fundamentals of Pure and Applied Economics, Volume 58 (John A. Ferejohn, ed.). Harwood Academic Publishers, Reading, United Kingdom. [217, 228]

Moulin, Hervé (1979), “Dominance solvable voting schemes.” Econometrica, 47, 13371351. [217] 
Moulin, Hervé (1986), “Choosing from a tournament.” Social Choice and Welfare, 3, 271291. [214]

Rasch, Bjørn E. (2000), "Parliamentary floor voting procedures and agenda setting in Europe.” Legislative Studies Quarterly, 25, 3-23. [212]

Shepsle, Kenneth A. and Barry R. Weingast (1984), "Uncovered sets and sophisticated voting outcomes with implications for agenda institutions." American Journal of Political Science, 28, 49-74. [214, 217, 230]

Vermeule, Adrian (2005), “Submajority rules: Forcing accountability upon majorities.” The Journal of Political Philosophy, 13, 74-98. [212]

Co-editor Nicola Persico handled this manuscript.

Manuscript received 9 March, 2015; final version accepted 22 December, 2015; available online 1 February, 2016. 